Portland State University

PDXScholar

6-13-1996

\title{
An Evaluation of English Spoken Fluency of Thai Graduate Students in the United States
}

Sugunya Ruangjaroon

Portland State University

Follow this and additional works at: https://pdxscholar.library.pdx.edu/open_access_etds

Part of the Bilingual, Multilingual, and Multicultural Education Commons Let us know how access to this document benefits you.

\section{Recommended Citation}

Ruangjaroon, Sugunya, "An Evaluation of English Spoken Fluency of Thai Graduate Students in the United States" (1996). Dissertations and Theses. Paper 5297.

https://doi.org/10.15760/etd.7169

This Thesis is brought to you for free and open access. It has been accepted for inclusion in Dissertations and Theses by an authorized administrator of PDXScholar. Please contact us if we can make this document more accessible: pdxscholar@pdx.edu. 


\section{THESIS APPROVAL}

The abstract and thesis of Sugunya Ruangjaroon for the Master of Arts degree in

Teaching English to Speakers of Other Languages were presented June 13, 1996, and accepted by the thesis committee and the department.

COMMITTEE APPROVALS:

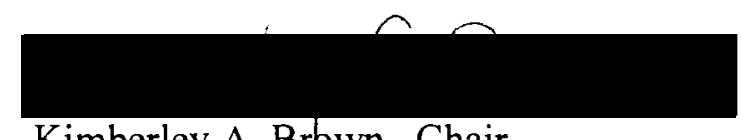

Kimberley A. Brpwn, Chair
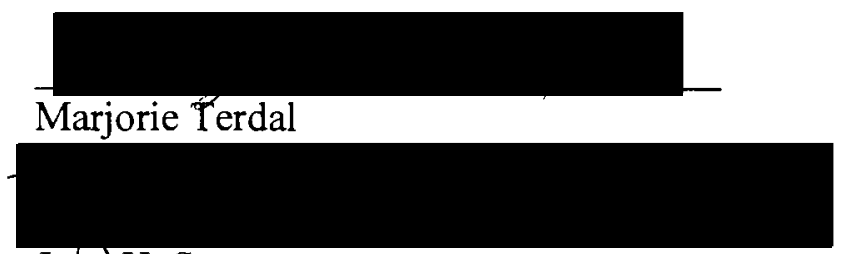

Joan H. Strouse

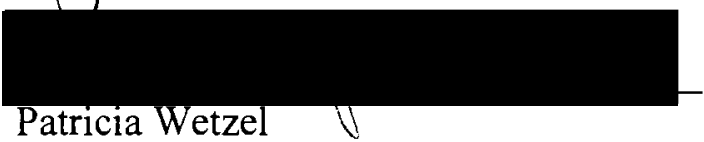

Representative Office of Graduate Studies

DEPARTMENT APPROVAL:

Beatrice Oshika, Chair

Department of Applied Linguistic

ACCEPTED FOR PORTLAND STATE UNIVERSITY BY THE LIBRARY

by

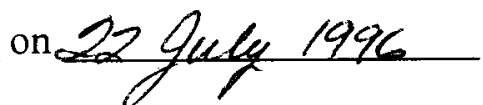




\begin{abstract}
An abstract of the thesis of Sugunya Ruangjaroon for the Master of Arts in Teaching English to Speakers of Other Languages presented June 13, 1996.
\end{abstract}

Title: An Evaluation of English Spoken Fluency of Thai Graduate Students in the United States.

Fluency is generally recognized as speaking with a high rate of speech. This study provides a greater understanding of the notion of fluency that speech rate is not the only measure to determine the fluency level of a speaker. Particularly in a second language (L2), fluency involves other features and it can reflect non-native speakers' capacity in using L2. Fluency is comprised of the continuity and the smoothness of speech without a high occurrence of hesitation phenomena and repair mechanisms.

The purpose of the present study was to analyze English spoken fluency of Thai graduate students. This study sought to answer the following questions: 1) How many of the intended messages from Thai graduate students are understood by English native speakers (NSs)? 2) To what factors do Thai graduate students attribute their fluency related behaviors? and 3) How orally fluent are Thai graduate students in the production of English?

Twenty Thai graduate students at a large university in the western United States volunteered as subjects. Both qualitative and quantitative analyses were used in the study. One-tailed t-tests were computed to examine the first question. 
The tests were considered significant at the .05 level. A highly significant difference was found confirming that listeners understand approximately $80 \%$ (2.4) or less of the subjects' messages due to errors in pronunciation, grammar, fluency, or vocabulary that occasionally interfere with intelligibility.

Retrospection of the subjects on the recorded conversations between the subjects and the NS interviewers answered the second question. The subjects reported that the main problem was a lack of vocabulary that significantly influenced the fluency level of their spoken English.

Hesitation, repair and rate of speech during the interview were counted as the characteristics of fluency and were used as parameters to determine the subjects' fluency and lack of fluency. The raw frequencies showed that there were 2 subjects rated as fluent and 18 subjects as non-fluent. To strengthen the validity of the third question, two-tailed t-tests and Mann-Whitney U-Wilcoxon Rank Sum W test were used to determine if a statistically significant difference existed between fast versus slow speakers. 
AN EVALUATION OF ENGLISH SPOKEN

FLUENCY OF THAI GRADUATE STUDENTS

IN THE UNITED STATES

by

SUGUNYA RUANGJAROON

A thesis submitted in partial fulfillment of the requirement for the degree of

MASTER OF ARTS

in

Teaching English to Speakers

of Other Languages

Portland State University

1996 


\section{ACKNOWLEDGEMENTS}

I would like to thank Dr. Kimberley Brown. Dr. Marjorie Terdal and Dr. Joan Strouse for advice, patience, understanding, and professionalism throughout the work. Deepest gratitude from all of my heart goes to Nisit Tantavichet, for his support and love throughout these tough years. My perserverance and a strong determination will not have endured long enough to complete the thesis without my beloved mother and sister who never stopped believing in me.

I am also indebted to Neal Loper who contributed his valuable time proofreading the whole thesis. Moreover, I owe big gratitude to people like Pamela Connors. Vidya Rangachari and others who are not named here at the Writing Center in Cramer Hall who helped me with writing. A special thanks to Mary Jane Bagwell for interviewing the subjects and also to Mary Jane Dresser, Catherine Berton and Jennifer Youngsook Sah for their assistance in evaluating the subjects' $L 2$ speech production. I would like to contribute my thanks to all of the Thai graduate student participants particularly Siriporn Pipatsattayanuwong for her hospitality. Also, a big thanks goes to Khun Sermpong Soonthronpong for his consistent kindness. A special word of thanks goes to my Thai friends who gave me encouragement and sympathy; I will never forget their friendship. The help I received from Sawako Yoshikawa and her boyfriend will be remembered as well. 


\section{TABLE OF CONTENTS}

PAGE

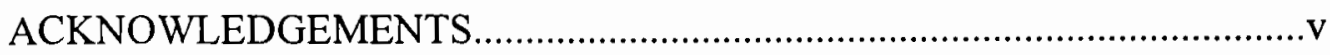

LIST OF TABLES ......................................................................................

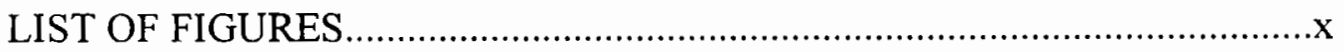

CHAPTER

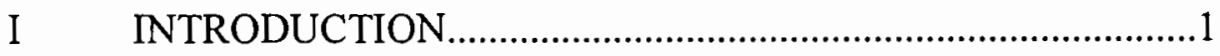

Statement of the Problem......................................................

Purpose of the Study ...........................................................

Research Questions............................................................

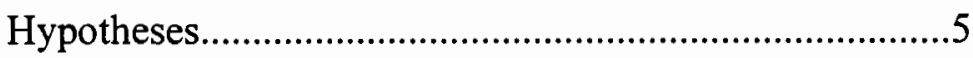

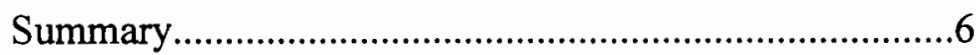

II REVIEW OF THE LITERATURE..........................................

Notion of Fluency.............................................................

Dimension of Fluency.......................................................

Hesitation Phenomena.....................................................

Repair Mechanism...........................................................12

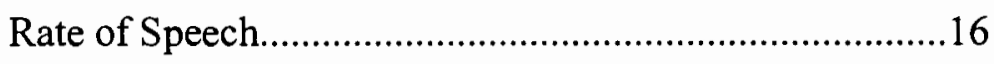

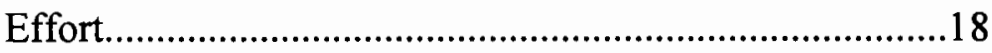

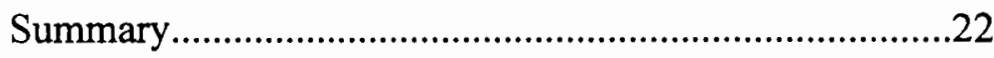

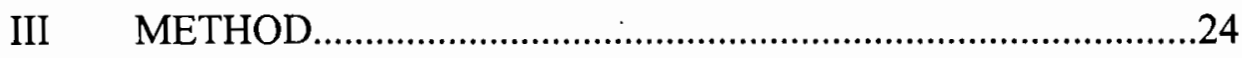

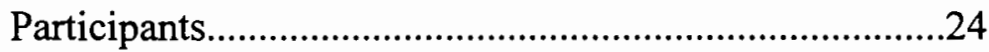

Subjects Analysis..............................................24

NS Participants......................................................27

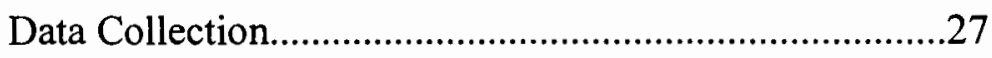

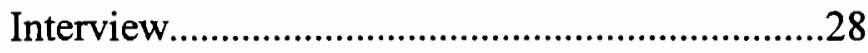

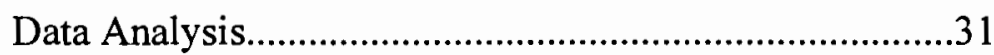

The Combination of Qualitative and

Quantitative Analyses............................................31 
Validity and Reliability of the Data Analysis..................44

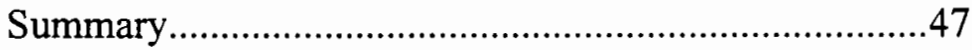

IV RESULTS AND DISCUSSION...........................................

Discussion of the Findings................................................50

Less Than $80 \%$ of Subjects' Messages

Understood by NSs..............................................50

Subjects' Encoding Capacity ParticularlySpeech

in Lexis Influences the Fluency Level of

Their Spoken English

Summaries of Means and Standard Deviations of

Fluency and Non-Fluency Behaviors..................62

Statistical Methods.................................................68

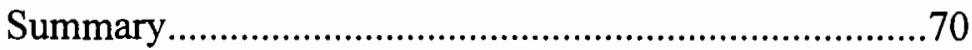

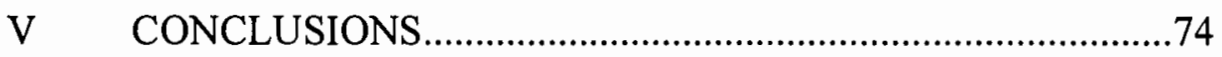

Summary of The Study........................................74

Implication for Thai Graduate Students in the United States........................................75

Strategies in Developing Fluency.......................77

Limitation and Methodological

Problems.....................................80

Suggestions for Further Research..................................81

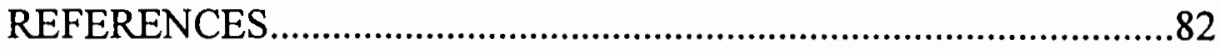

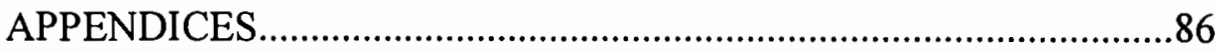

A DEFINITION OF EVALUATION SCORES.................86

B PROCEDURES OF COUNTING (SEMANTIC)

WORDS OR PHRASES................................................8

C SAMPLES OF TRANSCRIPTS .................................90

D BREAKDOWN BY SUBGROUP...............................101

E A SAMPLE BROCHURE..........................................107

F A WORKSHOP FOR ESL INSTRUCTORS: 
TEACHING FLUENCY.............................................111 


\section{LIST OF TABLES}

TABLE

PAGE

I Demographic Data of Thai Graduate Subjects.......................26

II The First Evaluator Scores ……………….........................51

III The Second Evaluator Scores................................................52

IV Frequencies of the Occurences of Fluency-Related

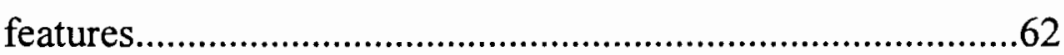

V Summaries of Means and Standard Deviations of

Fluency and Non-fluency Behaviors.........................................64

VI Fast Group Versus Slow Group:

Hesitation, Repair, the Sum of Hesitation and

Repair, The Speech Rate..............................................68

VII Breakdown in the Production Process

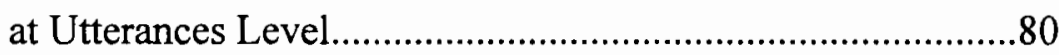




\section{LIST OF FIGURES}

FIGURE PAGE

1. ITA Test Score Sheet...............................................................33

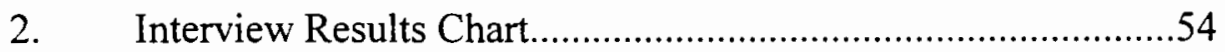




\section{CHAPTER I}

\section{INTRODUCTION}

Even though universities in Thailand offer an adequate number of graduate programs, due to the strong emphasis on English in Thailand (e.g. English is mandatory from primary school on (Kurian, 1992), many Thai graduate students come to the United States not only for the formal education but also the chance to expose themselves to English on a day to day basis. However, it has been my observation that my fellow students and Thai friends and colleagues who have returned home do not feel at ease in expressing their thoughts or producing them in English automatically. They all say that during the time in the U.S., Thai graduate students spend most of the time studying and have little expa universityre to social interaction with English-native speakers (NSs). They do not actually take opportunities in speaking English nor experience a full range of natural contacts with the American host. In their free time, they cling to their own members and speak their own language, rather than interacting with the members of the target culture. For these reasons, Thai graduate students appear to minimize their chances in receiving the input needed for second language (L2) development. As a result, insufficient practice in speaking English hinders Thai graduate students' L2 fluency.

Fluency can be defined as speaking with ease--a fluent speaker does not need to spend much thought or energy on L2 production, but rather, produces words that are 
strung together within utterances with nonfunctional pauses in a smooth fashion (Starkweather, 1987; Gelderen, 1991; Riggenbach, 1991). The concept of fluency is not well understood. The cut off points between perceived fluency and lack of fluency are not consistently agreed upon by researchers. Many researchers (i.e. Rehbein, 1987;

Riggenbach, 1990; Lennon, 1990; Arevart \& Nation, 1991; Gelderen, 1994 ) have suggested continuity of speech, rate of speech and amount of effort as measures of fluency. These researchers have examined spoken fluency of non-native speakers (NNSs) by counting the length and positioning of their silent pauses, the length of fluent speech that runs between pauses, the frequency and distribution of filled pauses, and the frequency of repetitions and self corrections in judging their fluency level. Mostly, studies of NNSs fluency use elicited data such as story telling based on a series of pictures, or delivery of speech as monologue. Few attempts have been made to provide empirical studies of NNSs' natural speech, unplanned / spontaneous conversation, by examining their fluency from naturalistic data. Few studies have examined discontinuities of information such as long silent pauses, frequent occurrence of filled and unfilled pauses, and slower speech than an expected rate. These elements need to be looked at because they could affect listeners' understanding.

\section{STATEMENT OF THE PROBLEM}

Most of the well-known proficiency tests (i.e.Test of English as a Foreign language (TOEFL) and the Michigan Test of English Language Proficiency (MTELP) basically measure learners' knowledge of the proper usage of English but not their ability 
to use the language verbally (Carroll, 1980). High TOEFL or MTELP scores cannot predict students' oral English proficiency because neither the TOEFL nor the MTELP measure oral language proficiency but instead, written linguistic fluency. The verbal fluency of NNSs has long been recognized as a crucial factor in judging target language proficiency. My initial interest in spoken English fluency arose from my experience, and that of my fellow students and Thai friends and colleagues. We speak with pauses, hesitations, false starts, repetitions, a smaller number of semantic words per minute and a large amount of effort. The frequent occurrences of these behaviors are perceived as nonfluency markers owing to their interference with the intended messages. Moreover, these features result in reducing listeners' comprehension since these interruptions are not only the causes of the discontinuities of information but also distract the listener from the message.

I have observed that my Thai colleagues and I sometimes have difficulty expressing in English what we are completely capable of producing in Thai. When we are unable to express an idea in English, we frequently abandon the message or produce the message along with non-fluency related features in our utterances. As Faerch and Kasper, 1980 point out, the grammatical rules for combining smaller units into larger structures of the second language to produce novel or unfamiliar utterances may belong to the language user's system but the user fails to apply it fully for some reasons. Fillmore (1979) also explains that if a speaker is not syntactically fluent, semantically fluent, and pragmatically fluent, he is likely to be less fluent in his speech production 
since his ability in encoding highly complex sentences is poorer than that of fluent speakers. In addition, the speaker masters less choice of vocabulary and does not know how to express his ideas under a wide variety of social circumstances.

\section{PURPOSE OF THE STUDY}

This study analyzes oral fluency of Thai graduate students in the United States. First, the study investigates English native-speakers' perception of the intelligibility of Thai graduate students' messages. Second, it examines the speakers' reasons for hesitation and restart behaviors that may or may not occur during the interview. Third, the study measures the fluency level of their actual speech production. The three fluency features measured are: 1) hesitation or discontinuity phenomena, 2) repair phenomena (repetition and restart), and 3) rate of speech. The methodological frame for this analysis is partially guided by Riggenbach (1991).

\section{Operational Definitions}

Hesitation in this study is defined as:

- Unfilled pauses - a silence of 1 second or greater

- Filled pauses - voiced "fillers," which do not normally contribute additional lexical information

- Nonlexical ("um," "um") - fillers that are not recognized as semantic words because they contain little or no semantic information

- Lexical ("you know," "I mean") - fillers that are recognized as words but in context contribute little or no semantic information

Repair in this study is defined as:

- Retraced restart - reformulations in which part of the original utterance is repeated 
- Repetition - exact adjacent repeats of sounds, syllables, words, or phrases

- Insertion - a retraced restart in which new (unretraced lexical items are added)

- Unretraced restart - reformulation in which the original utterance is rejected (=false start)

Rate of speech is defined as:

- Total number of words per minute - the count for all words including partial words (using the criterion that partial words contain not just an initial consonant but also a vowel and thus are recognizable as words)

- Semantic number of words per minute - the count for words that contain semantic information excluding filled/unfilled pauses and retraced/unretraced restart.

\section{RESEARCH QUESTIONS / HYPOTHESES}

Three hypotheses about fluency in English follow from three research questions:

1) How many of the intended messages in the corpus analyzed from Thai graduate students are understood by English-native speakers?

Hypothesis 1: Listeners will understand approximately $80 \%$ or less of subjects' messages due to some errors in pronunciation, grammar, fluency, and vocabulary that occasionally interfere with intelligibility.

2) To what factors do Thai graduate students attribute their fluency related behaviors?

Hypothesis 2: Speakers' encoding capacity particularly in lexis significantly influences the fluency level of Thai graduate students.

3) How orally fluent are Thai graduate students in the production of English? 
Hypothesis 3: English-speaking Thai graduate students in this study are unable to simultaneously plan utterances and produce them in a smooth manner. ("Utterances" are defined as spoken words forming the complete expression of a thought and used to express communicative intentions (Bamford, 1989).

\section{SUMMARY}

This research will investigate the English oral fluency of Thai graduate students by looking at their actual speech production as measured by the fluency related features of 1) hesitation (discontinuity); 2) repair mechanism; and 3) rate of speech. It will incorporate both qualitative and quantitative methods of analysis by examining not only native speakers' rating but also subjects' reasons in the case of speaking hesitancy and lack of fluidity in L2 in an interview situation. 


\section{CHAPTER II \\ REVIEW OF THE LITERATURE}

The literature review will discuss the characteristics of speech production which are perceived as fluent and those which are perceived as non-fluent by many researchers, namely, Starkweather, 1987; Riggenbach, 1991; and Schmidt, 1992. Because the notion of fluency and its characteristics vary from scholar to scholar, it is necessary to understand the points of view of these researchers.

Notion of Fluency: Speech Production

It is usually mentioned first by researchers (i.e. Hieke, 1985; Schmidt, 1992; Starkweather, 1987 ) that the concept of fluency is not well understood because there seem to be two meanings of fluency. Lennon (1990) distinguishes those meanings as broad and narrow. In its broad sense, fluency is defined as the global ability of speaking a foreign language. This broad sense of fluency is used for employment, study abroad purposes, filling out academic forms, or as a mark of social accomplishment. (Schmidt, 1992) gives an example of someone saying, "my friend speaks four languages fluently." In this case, fluently means to speak well; however, the meaning of "well" is not clarified.

Fluency in a narrow sense, according to Lennon (1990), is similar to the way in which other researchers have defined fluency. Schmidt considers fluency as the skill of a 
person who unconsciously performs an action to speak a language rather than primarily knowing the concept of syntax. Fluency, therefore, is a concept that is seldom mentioned formally in a classroom nor does it appear as a test objective (Hieke, 1985). According to various researchers (Arevart \& Nation, 1991; Gelderen, 1994; Lennon, 1990; Rehbein, 1987; Riggenbach, 1991; Schmidt, 1992; Starkweather, 1987) fluency is speaking with ease--a fluent speaker does not need to spend much thought or energy on it. Rather he/she produces words that are strung together within utterances with nonfunctional pauses in a smooth fashion (Starkweather, 1987; Gelderen, 1991; Riggenbach, 1991). However, Sajavaara and Lehtonen (1980) point out that the concept of fluency is still ambiguous. For example, how many words per minute does the speaker need to produce to fit into the parameter of fluency? Or how many "correct" pauses in speech production would be counted as typical of fluent speech? Genre, such as sport commentary or a Christmas sermon, can effect the rate of speech so that makes it difficult to determine. Sajavaara and Lehtonen explain that measuring fluency from the actual message alone is not sufficient to define the parameter of fluency because some people are fluent but still unintelligible to hearers while others, who are considered non-fluent, are able to communicate rather well. Both linguistic acceptability and smooth continuity of speech comprise fluency (Sajavaara \& Lehtonen, 1980). In order to rate non-native speakers' speech, it is vital to control these variables mentioned above. 
Dimensions of Speech Fluency

Fluency is a multidimensional behavior which mainly consists of three categories: continuity or smoothness of speech (hesitation and repair phenomena), the rate of speech and the effort a speaker makes in his speech production (Starkweather, 1981). According to the researchers, NNSs who are perceived as being fluent will be expected to produce utterances smoothly, unhesitatingly and effortlessly at an expected rate after they begin speaking.

Continuity

Continuity is the first element of speech fluency. Continuous speech flows without the frequent occurrence of hesitation phenomena and repair mechanism that may disturb the overall impression of fluency.

\section{Hesitation Phenomena}

Hesitation phenomena are described as pauses, and pauses are usually divided into unfilled and filled. The unfilled pause is simply a silence of .5 second or greater (Riggenbach 1991). The filled pauses are utterances that do not contribute any lexical meaning such as "um," "uh," "ah," "er," and the like. Short pauses of .4 second or less occur in normal speech and are not considered a signal for lack of fluency. The feature distinction that Riggenbach (1991) points out between fluent and non fluent pauses is that fluent-sounding short pauses are produced at clause boundaries; in contrast, non-fluent sounding short pauses usually occur in midclause or midphrase but not at clause 
boundaries (juncture and hesitation pauses). The non-fluent pauses, described as choppysounding, are more likely to interrupt the flow of the message.

One way to measure people's lack of skill with the target language is through the frequency of those idiosyncratic interruptions. For example, people who have uncertainty in linguistic knowledge will tend to produce idiosyncratic pauses when asked to speak with spontaneity (Starkweather, 1987). In contrast, if they are required to read aloud, they will demonstrate only conventional pauses, occurring at syntactic boundaries (Starkweather 1987). But it is not always the case that idiosyncratic pauses will function as an aspect of the performance of a person who struggles finding word choices, or has syntactic difficulty while formulating his thoughts and language all the time.

Fathman (1980) reports a case of young second language users who often have pauses before a content word in their speech. The pauses probably imply that they are having lexical searching problems. The more syntactically complex the sentence is, the more pauses are likely to be produced. It has been found that there is a significant correlation between pauses and uncertainty of how to construct sentences. In researching these pauses, Lennon (1990) has drawn attention to what is called communication strategy. She states that it might be difficult to determine the cut-off points of pauses and hesitation as to whether they act as useful tools to help sustain the communication or as markers of the system breaking down. Nonetheless, a major determinant of judgments of fluency is how the information flows. This criterion was used in this study in evaluating the fluency of Thai graduate students' speech. 
Ioup, Boustagui, Tigi and Moselle (1994) report that the subject of their study is rated as fluent as a native speaker of Arabic because she is capable of manipulating Egyptian Arabic discourse markers and pause fillers and applying them appropriately to the situation. As Sajavaara and Lehtonen (1980) mention, "to be fluent in the right way, the speaker has to know how to hesitate, how to be silent, how to self-correct, how to interrupt, and how to complete his expression" (p.71). The fluent speaker demonstrates use of conventionalized language and knows that pauses are useful tools in communication.

Riggenbach (1991) concludes from her study that the distinguishing marks of non-fluent speech are in this order of priority of importance and degree of salience:

1) "Frequency, placement, and degree of chunking, and type of filled and unfilled pauses"

2) "Rate of speech"

3) "Frequency and the function of repair" (p.438, 439).

Based on Riggenbach's study, the above markers of non-fluent speech will be used to evaluate the subjects' speech in this study, for example; "How many unfilled pauses do the subjects produce in the middle of phrases?" The purpose is to investigate my third research hypothesis that English-speaking Thai graduate students are unable to simultaneously plan utterances and produce them in a smooth manner.

\section{Repair Mechanism}


Besides hesitation phenomena, the repair mechanism is also regarded to be another marker that interrupts the flow of messages. Repair in the production of speech is classified into two types: covert pre-execution and overt post-execution plan shift (Faerch \& Kasper, 1983, p.13). Overt post execution can be detected but covert pre-execution cannot because speakers correct their mistakes before speaking them. Overt postexecution occurs when speakers execute a plan and then find out that the plan does not match with the established goal. They then decide to change or repair the plan which has already been uttered. This study examines only overt post-execution. The features of the repair mechanism are composed of "repetition of sounds, syllables, words and phrases, prolongations of sounds, parenthetical remarks such as 'you know', and false starts as in 'I am going to, I've got to leave now'” (Starkweather, 1987, p. 21). Similarly, Riggenbach (1991) defines these repair mechanisms as retraced restart and unretraced restart. Retraced restart is "reformulation in which part of the original utterance is repeated" (p.427). Retraced restart consists of repetition and insertion. Repetition is "exact adjacent repeats of sounds, syllables, words, or phrases" (p.427), whereas, insertion is "a retraced restart in which new/unretraced lexical items are added" (p.427). An unretraced restart is a false start. The emergence of false start, incomplete clauses, or retraced restarts might indicate that there are syntactic planning problems. These characterizations will be used in the base of this study. Also, Lennon in his instrument investigation of fluency (1990) defines repair mechanism as a vocal dysfluency marker component. Like Riggenbach, Lennon examined repetitions, self-corrections, filled 
pauses and a percent of repeated and self-corrected words. Rehbein (1987) used different terms but examined similar features. They included "pause," "em," "eh," "first effort," "self-interrupt," "improved version and new start" (p.101). The phenomena identified above will make up the category examined for this study.

Generally, repetitions exist in the shape of repeats of one or two words followed by the completion of an utterance. The occurrences of repeats often follow the first or second function word of a constituent beginning an utterance (Fathman, 1980). Fathman gave examples of common repetitions like "then he...then he started to cry", or "and the boy...the boy got...got hot...hot" (p.79). According to Fathman (1980), these repetitions arise from speakers giving themselves time to plan what to utter next. These repeats reflect the speakers' ability in lexicon retrieve and syntactic organization in structuring their sentences. On the other hand, second language learners may use the repair mechanism as a device to counterbalance non-fluency while communicating. In other words, repair mechanisms serve a useful communication function. Using such repair mechanisms assists in maintaining the conversation between NNSs and NSs in that the interlocutors will not turn away from the speakers' messages, nor interrupt them (Rehbein, 1987).

Even though in many studies self-correction is not a distinguishing marker of nonfluency features, this study defines self-correction as insertion and unretraced restarts. Thus, it is essential to understand why L2 speakers produce self-correction and how it 
affects speech fluency. Levelt (1983) discusses in his study that spontaneous self-repair is the effect of the monitoring process of speakers toward their own speech production which may interrupt the smoothness and continuity of the messages. There are two theories of self-corrections defined as "the production theory of monitoring" and "the perceptual theory of monitoring" (Levelt, 1983). When the speakers detect their mistakes, they will correct them. Self-correction in speech occurs from a complex interplay between perceptual and productive processes (Levelt, 1983). First, speakers must be capable of detecting an error or inappropriateness in their production. Second, speakers decide to interrupt their own speech and start to correct the original utterances to new utterances. Levelt (1983) notes that "the speaker will, in some way, 'transfer' structural properties of the original utterance to the correction" (p. 42) [and] "by transferring and reusing structural properties of previous speech the speaker may at the same time gain in fluency, and establish discourse coherence to the advantage of the listener." (p. 42) Fathman (1980) suggests that most self-corrections involve speakers' encoding capacity of lexis in such a way that the speakers will substitute one word for another as soon as they realize that they provide listeners incorrect information and decide to correct themselves. Another possibility is that the speakers realize that they are failing to express in L2 what they wish in L1. In Fathman's (1980) study, the subjects were more concerned about providing correct or sufficient information rather than correct grammatical forms. Basically, it is still undecided whether or not self-correction is a marker of non-fluent speech. As noted earlier, in one point of view, self-correction 
reflects the speakers' knowledge of L2 structure. On the other hand, high occurrences of self-correction during speaking interrupt the flow of the carried messages.

Lennon's, (1990); and Riggenbach's, (1991) findings provide strong evidence supporting Levelt's (1983) idea that self-correction functions as a part of the fluency development of proficient speakers. Self-correction indicates the "speakers' increased ability to reformulate, monitor, and self-correct production on-line" (p.413). According to Lennon's (1990) research, it was not until the final week of his 6 month study that three of the four subjects showed an increase of self-corrections; they did, however, decrease repetition, and filled and unfilled pauses at boundaries in their speech production. The results suggest that fluent $\mathrm{L} 2$ learners are more capable of reformulating, monitoring and self-correcting their own production than non-fluent L2 speakers. Therefore, self-correction, in contrast, signals L2 speakers' fluency development, and is not a marker of non-fluency. However, this study examines self-correction as a marker of non-fluency when it accompanies one other fluency-related feature or a chunk of disfluencies. This is crucial because a chunk of disfluencies causes the loss of smoothness of speech production or causes it to be non-fluent sounding.

Similarly to Lennon, (1990); Riggenbach, (1991) found that one of her subjects was not apt to detect her grammatically incorrect usage in her speech. She, nevertheless, produced messages without interruption, along with a relatively high rate of speech and a relatively low pause count. Despite her high rate of speech and low pause count, she was rated as a non-fluent speaker as she had little awareness of what comprised structurally 
appropriate English. My study aims at investigating the characteristics of a non-fluent speaker by counting hesitation, repairs and rate of speech not grammaticality. Gelderen's (1994) findings suggest that long-lasting pauses and hesitation or high frequency of repeats, exclamation and correction all have stronger relations with fluency ratings than short-lasting pauses or low frequency of occurrences of these behaviors. In conclusion, if the listeners perceive speakers using a great amount of effort in their production mechanisms via hesitation phenomena and repair mechanism, they will judge the speakers to be non-fluent.

Before discussing another element of fluency, it is important to realize that people who can perform their L2 with facility must have sufficient syntactic, semantic, pragmatic, and phonological knowledge of the target language. Also, in order to be perceived as fluent, NNSs should master a large vocabulary. Most speakers of a given language express their ideas more easily and directly as they obtain a better grasp on its vocabulary and are presented with a wider range of word choices. Nevertheless, not every L2 learner can achieve this linguistic repertoire. Different speakers have varying command of vocabulary (Fillmore, 1979). It is common that L2 learners' oral proficiency will differ from one to another to some degree.

\section{Rate of Speech}

In addition to continuity of speech, the second aspect of fluency is speech rate. Riggenbach (1991) measures the "amount of speech" by counting "all semantic units including filled pauses and partial words (using the criterion that partial words contain 
not just an initial consonant but also a vowel and thus are recognizable as words)" (p.428). Other researchers usually look at the number of syllables per minute or syllables per second (syll/sec) In addition, there are two variables that always play a modifying role with the speech rate: speech mode and type of delivery (Hieke, 1985). Speech mode is exemplified as "reading aloud, reciting, paraphrasing, lecturing" and type of delivery as "casual, deliberate, allegrissimo" (Hieke, 1985, p.139). As mentioned earlier, speech rate is the most objective measurement if comparable to pauses, repair or parenthetical marks ("well, you know, in other words") since "it is capable of [not only] differentiating native from non-native speech" but also intermediate from advanced language proficiency levels (Hieke, 1985, p.139).

Riggenbach's (1991) study shows significant differences of speech rate between fluent and non-fluent NNSs. According to a quantitative method of analysis, a subject might appear to fall into a group of high fluent speakers in terms of her rate and amount of speech. The raters, however, put her in the group of low-fluent speakers by giving the reason as her inability to produce grammatically correct form in her utterances. By qualitative methods of analysis, fluency is not restricted only to these three features-hesitation, repair, and rate and amount of speech.

According to Starkweather (1987), a common superficial measurement used to judge the fluency level of second language speakers is how fast they speak. For instance, if the same speaker delivers the same passage but at different speech rates, it is not always the case that the faster speech will be perceived as the more fluent one. Different groups 
of people might reach different conclusions, as one group weighs discontinuity heavily while another group stresses words per minute (wpm). It should be noted that filled pauses and hesitations such as "it's like you know," "what I mean, what I mean is," I don't know how to say this word," are counted as semantically empty words. Thus, the fluency level of two passages produced at the same rate, with varying amounts of information will be judged differently. The amount of information conveyed rather than the rate or continuity of speech production is the key element of how fluent a second language speaker will be perceived.

\section{Effort}

Another significant characteristic of fluency is effort, in that the fluent speaker is able to speak the target language with little thought. It is common that second language learners are not able to produce the target language as effortlessly as native speakers do. The amount of effort in using L2 varies from one speaker to another to some degree. It depends on how well L2 speakers can automatize their speech production. The more people automatize, the less effort they put in. Due to the fact that the mechanism of internalization of the language between NSs and NNSs is different, Sajavaara (1987) points out that second language learners are able to be fluent in their speech production by internalizing a language based on developmental stages in naturalistic L2 acquisition.

Naturalistic L2 acquisition is explained in Clahsen's (1987) study as, when learning a language, there are similarities between $\mathrm{L} 1$ and $\mathrm{L} 2$ acquisitional processes leading NNSs to fluency in speech production in untutored/ natural settings. In his 
research, he finds that both NSs and NNSs of German learn word order rules in the same way by strictly following ordered developmental sequences. However, there appear to be differences in some stages in their acquisition. For example, in their speech production, with respect to some syntactic rules, $\mathrm{L} 1$ learners start out with a "more general hypothesis and a specific assumption about German grammar (Clahsen, 1987, p. 71). Even though L1 and L2 learners apply different hypotheses about the structure of the target language, both of them eventually are capable of internalizing knowledge about German word order. Clahsen (1987), in addition, finds that the "discontinuous word order and inversion" of German seems to present difficult and complicated learning tasks for not only NNSs but also NSs. Consequently, during the acquisition of complex linguistic operations, learners make use of strategies of simplification which assist them in internalizing their linguistic repertoire. It will take some time for L2 learners to acquire inversion's rules. To make the learning process easier, the complexity of the task should be diminished by decreasing the number of contexts until learners have internalized "inversion," thus, strategies of simplification will not be necessary any more. These two learning processes, the integration of new linguistic knowledge with previously acquired knowledge, and strategies of simplification, can lead non-native speakers to internalization of the target language. From this stand point, one could argue that a speaker who produces speech which is perceived as lacking fluency features has not "automatized the required linguistic operation" (Sajavaara, 1987). 
Starkweather (1987) regards the effort that goes into speech production as a premise since there is no empirical way that enables listeners to measure the amount of effort of speakers while producing L2. The amount of time formulation and transmittal of a given amount of information, nevertheless, bears pertinent information about the amount of effort that speakers devote to the production of speech.

Effort has been recognized in two distinct ways: effort of thought and effort of muscle (which will not be discussed here) (Starkweather, 1987). Fluent speech requires little thought and little muscular exertion from the speakers. In speaking fluently, people tend to focus on content rather than on the mechanism of linguistic communication. Nevertheless, a non-fluent speaker uses an inordinant amount of mental energy merely in forming sentences in the target language. Starkweather (1987) compares the process of fluent speech production with the breathing system of human beings stating that "it goes on without any conscious effort but can be consciously controlled should the occasion demand it" (p.38). Therefore, speech production is regarded as an "automatic or semiautomatic behavior" (Starkweather , 1987, p.38) or automatic procedural skill (Schmidt, 1992, p.358).

In the case that planning and uttering a thought does not happen simultaneously, then the oral production will not be produced in a fluent manner and will tend to interfere with the flow of information. The planning of an utterance and the execution of it are defined as two different kinds of behaviors: [planning is more a conscious, voluntary 
behavior and takes only a small proportion of the total time while execution consumes more time than automatic] (Starkweather, 1987, p.40).

The duration of pauses seems to be a measure of the amount of effort that goes into planning the subsequent utterance. The location of the pauses will serve as a distinguisher between pause time that is being used to plan for the production of speech sounds or to plan the structure of sentences. As mentioned earlier, pauses will occur in different positions; either at clause boundaries or within the clause. Being in a different speech mode situation, reading aloud versus speaking spontaneously, L2 learners produce pauses in different locations. Regarding speakers who pause within the clause, it is suggested that they interrupt themselves to think about what words they are going to use, or how to structure the rest of the sentence. In contrast for the speakers who pause at the clause boundaries, it is indicated that they already knew how the sentence is going to be structured and what words they are going to use but they just think about what they are going to say. In my study, the location of pauses within the clause is used as an indication of the subjects' fluency in speaking.

Based on psychological mechanisms underlying second language fluency, Schmidt (1992) discusses two different forms of processing: automatic and controlled processing. Automatic processing is characterized by an effortless speaker. On the other hand, controlled processes occur when a speaker applies skill to a new situation. Learners can develop skill from controlled to automatic processing by practice. Arevart and Nation's (1991) finding provides strong evidence of this idea. Their subjects were asked 
to tell the same story three times to different listeners reducing the time for each retelling of the story. Consequently, there was a distinct improvement on the subjects' speaking. The subjects apparently increased their speech rate with an increase of words per minute and also decreased the number of hesitations in the final delivery. This result suggests that learners become more automatic in producing the target language by means of repeating the same tasks. Practice appears to be indispensable for fluency in L2 which will prove to be strongly confirmed if the hypotheses in this study are supported.

Mclaughlin et al. (1983) see the close connection between fluency and automatic processing. They report that non-fluent and fluent speakers are different in their speech production because the degree of automatization of lexical processing are varied between them. Fluent L2 learners display their speech more with the properties of automatic processing in that they can produce smooth sentences at a normal rate. This will occur only when speaking mechanisms have been automatized (Rehbein, 1987; Sajavaara, 1987). Some dimensions of L2 fluency, namely, speech rate and the length of fluent runs between pauses correspond with automaticity; on the other hand, other elements of fluency may reflect the fact that all complicated learning tasks require both automatic and controlled processes, usually organized in a systematic network or hierarchy (Levelt, 1977; Schneider et al., 1984).

\section{SUMMARY}

This chapter reviewed three main elements of perceived fluency and non-fluency characteristics in second language speech production. Many empirical studies show that 
the distinguishing markers of fluent speech are that a speaker is able to produce smooth speech without the high occurrences of hesitation or repair, to produce speech at an expected rate, and also to produce speech with ease. These features are directly manifested in "fluency" performance.

This study will examine English spoken by Thai graduate students in the United States following the base of the review of the literature. It will analyze their actual speech production quantitatively by counting the occurrence of filled/unfilled pauses, retraced/unretraced restarts including total number of words and semantic words per minute, with semantic words per minute defined as words that contain semantic information excluding filled/unfilled pauses and retraced/unretraced restarts. 


\section{CHAPTER III \\ METHOD AND PROCEDURES}

The research methodology employed in the study is presented in this chapter. To examine English spoken fluency of Thai graduate students in the United States, the data were collected by eliciting their spontaneous speech while having conversation with native English speakers. The participants' student status, ages, length of residence (LOR), and fields of study are the independent variables which are closely controlled. The dependent variable is the subjects' spoken fluency level.

\section{PARTICIPANTS}

\section{Subjects Analysis}

The primary subjects in this study were 20 Thai graduate students at a large university in the western United States. All were in the field of Science, Engineering, and Agriculture from various departments such as Food Science and Technology, Industrial Engineering, Horticulture etc. (see Table I). Their length of residence in the Pacific Northwest ranged from 10 weeks to 3 years and 4 months. Their ages are between 23 to 36. Their Test of English as a Foreign Language (TOEFL) scores ranged from 520 to 597. Moreover, none were ever exposed to English in their daily lives before coming to the Northwest except one of the subjects (See Table I: subject T4). While she was a sophomore student, she decided to come to study in the United States for a year. She later 
went back to Thailand; however, she received her bachelor's degree from a U.S. university.

This subject group was selected for a number of reasons. First, all were graduate students focusing on disciplines which require a significant volume of assignments, problems solving tasks and lab testing. International students tend to be reserved as practicing speaking English relates to class participation and class participation is not obligatory in these majors. Furthermore, these participants spent most of their time with Thai colleagues, studying within their Thai group, generally maintaining close social interactions with other Thai students. This clannishness hinders their exposure to native English speakers and the English speaking community, resulting in fewer opportunities to practice speaking English.

Ratio of utterances to opportunities to speak per day (see Table I) was provided by the subjects in a self-evaluation of their L2 production. Only a small proportion of the group of twenty subjects scored their L2 output and production high. Only one subject rated herself in the upper category for L2 production, principally because she spent most of her time in the research lab working around NSs.

Second, all are 23 or older and considered adults. As an adult starter, it may be difficult for them to become communicative fluently in L2. According to LarsonFreeman and Long (1991), they say that "...younger is better in the most crucial area, ultimate attainment, with only quite young (child) starters being able to achieve accentfree, native-like performance in a SL" (p. 155). 
Third, their length of residence seems to affect their fluency in such a way that the longer they are in an SL environment, the better they speak English. The increased fluency by NNSs who stay longer in an English speaking environment is likely the result of active listening to media such as television and radio, reading books and newspaper, and listening to class lectures as well as interacting with other English speaking students. NNSs may increase English vocabulary and expressions simply from their reception and apply it to their speech production.

TABLE I

DEMOGRAPHIC DATA OF THAI GRADUATE SUBJECTS

\begin{tabular}{|c|c|c|c|c|c|c|}
\hline Subject & Sex & Age & $\begin{array}{l}\text { Length of } \\
\text { Stay in USA }\end{array}$ & Field of Study & $\begin{array}{c}\text { The latest } \\
\text { TOEFL } \\
\text { scores }\end{array}$ & $\begin{array}{c}\text { Ratio of } \\
\text { utterances } \\
\text { to opportunities }\end{array}$ \\
\hline T1(Ph.d) & $F$ & 36 & 1YR 4 MO & Animal Science & 543 & $1: 10$ \\
\hline T2 (MS) & $F$ & 23 & $1 \mathrm{YR}$ & $\begin{array}{l}\text { Marine Resource Management } \\
\text { in Oceanography }\end{array}$ & 547 & $5: 10$ \\
\hline T3 (MS) & $M$ & 27 & $1 Y R$ & Chemical Engineering & 560 & $2: 10$ \\
\hline $\mathrm{T} 4(\mathrm{MS})$ & $F$ & 24 & $10 \mathrm{WK}$ & Environmental Engineering & 583 & $4: 10$ \\
\hline T5 (MS) & $F$ & 23 & $4 \mathrm{MO}$ & industrial Engineering & 577 & $1: 10$ \\
\hline T6 (MS) & $\bar{M}$ & 24 & $2 Y R$ & Industrial Engineering & 573 & $0.5: 10$ \\
\hline T7 (MS) & $\bar{M}$ & 23 & $7 \mathrm{MO}$ & Industrial Engineering & 543 & $1: 10$ \\
\hline T8 (MS) & $\bar{M}$ & 23 & 1 YR $5 \mathrm{MO}$ & Industrial Engineering & 597 & $4: 10$ \\
\hline T9 (MS) & $M$ & 26 & $1 Y R$ & Construction Engineering & 583 & $1: 10$ \\
\hline T10(MS) & $F$ & 24 & $3 Y R$ & Management Horticulture & 573 & $3: 10$ \\
\hline T11(MS) & $M$ & 23 & $9 \mathrm{MO}$ & Chemical Engineering & 567 & $2: 10$ \\
\hline T12(MS) & $M$ & 24 & $7 \mathrm{MO}$ & Chemical Engineering & 590 & $4: 10$ \\
\hline $\mathrm{T} 13(\mathrm{MS})$ & $M$ & 24 & $1 \mathrm{YR}$ & Industrial Engineering & 557 & $1: 10$ \\
\hline T14(MS) & $F$ & 24 & $2 Y R$ & Industrial Engineering & 573 & $2: 10$ \\
\hline T15(MS) & $\bar{M}$ & 24 & $1 \mathrm{YR} 6 \mathrm{MO}$ & Industrial Engineering & 520 & $1: 10$ \\
\hline $\mathrm{T16}(\mathrm{MS})$ & $\mathrm{M}$ & 25 & $2 Y R$ & Economic & 567 & $3: 10$ \\
\hline $\mathrm{T} 17(\mathrm{MS})$ & $\mathrm{F}$ & 24 & 1 YR $3 \mathrm{MO}$ & Environmenta! Engineering & 573 & $3: 10$ \\
\hline T18(MS) & $\bar{M}$ & 24 & $1 \mathrm{YR} 4 \mathrm{MO}$ & Mechanical Engineering & 553 & $1: 10$ \\
\hline T19(Ph.d) & $\mathrm{F}$ & 24 & 3 YR 4 MO & Food Science \& Technology & 560 & $9: 10$ \\
\hline T20(MS) & $F$ & 23 & $3 \mathrm{MO}$ & Chemical Engineering & 567 & $3: 10$ \\
\hline
\end{tabular}

* Ratio of utterances to opportunities $=$ ratio of actually speaking to opportunities to speak per day 


\section{NS Participants}

Four NS participants were involved in two parts of this study. For the research methodology, two native English speakers conducted interviews; one interviewed 12, another interviewed 8 Thai graduate students, each at a different scheduled time. It was the responsibility of NS participants to ask the subjects questions while they were having normal, friendly conversations with them. One NS participant has a Master's degree in Adult Education, with emphasis in English as a Second Language (ESL), in addition to being an ESL instructor at the English Language Institute (ELI) at a university in the Northwest Pacific. Her current educational pursuits include a $\mathrm{Ph} . \mathrm{D}$ in education at that university, focusing on ESL Second Language Acquisition and Intercultural Communication. Another NS participant has a Master's degree in TESOL from Queens College in New York city, and has taught ESL in New York and been an ESL instructor at the ELI at a university in the western United States.

For the data analysis part, there were two native English speakers participating as raters to evaluate the comprehension of the subjects' speech production. The results scored by them were used to answer the first specific research question of this study, "How many of the intended messages from Thai graduate students are understood by English-native speakers?" In this phase of the study, one NS participant recently received a TESOL certificate from a large university in the western United States, while the other 
has a Master's degree in TESOL from the same university, with 14 years teaching experience.

\section{DATA COLLECTION}

The research methodology employed in this study involved the interview between a NS and the subject. Data collected from these interviews were used as a means to assess the subjects' fluency in English. In conjunction, there was a retrospective interview in $\mathrm{L} 1$ between the researcher and subjects as to their perception of the reasons for speaking hesitancy and lack of fluidity in L2 during the interview with the NS. A pilot study showed that a monologue was not a good example of real communication since the subjects "ran out" of things to say and paused for an extended period of time before starting on a new topic. Since this action would confound this study of fluency, an interview format was used

\section{Interview}

This interview was semi-structured. The researcher compiled a list of questions, although it was left up to the NS participant interviewer to choose the order in which the questions were asked. Some flexibility was given to the interviewer to pose additional questions that followed the general concept of the study, and seek clarification when the subjects' answers were not comprehensible.

\section{Piloting the Interview Questions}

A pilot study was conducted with 6 subjects. In that instance, a NS interviewer asked a variety of questions of the subjects in $\mathrm{L} 1$. The questions covered a wide range of 
knowledge and personal interests, including Thai culture, tradition and religion, economics, politics, movies, population, environment, communication and transportation. Following this, the questions were reviewed and reduced from 80 to a core group of 12 , which ranged from personal matters to general and specific questions about the Kingdom of Thailand. The length of responses from the subjects in the pilot study determined which questions to eliminate. If the subjects provided a long answer to a question, that question was selected. On the other hand, if the subjects provided a short answer to a question, that question was eliminated. Also, every effort was made to eliminate questions which elicited anything other than a spontaneous response and those which required the subjects to struggle with the content. However, in daily life while in an L2 environment, NNSs use L2 to answer both familiar and unfamiliar questions. Thus, questions with difficult content were likely to occur, and NNSs somehow had to handle those types of questions.

The core group of 12 questions are as follow:

1. What is your name and what are you studying?

2. Why did you choose Oregon, not the other states, or not England or Australia?

3. How did you adjust your study habits when you started your first term?

4. What surprised you when you first came to the US?

5. Could you tell me about traditions in Thailand, for example, American has Halloween, Christmas day, Valentine's day. How about in Thailand? Explain about that and its meaning.

6. Is there any corruption in your country? What do you think about it, and how can you help solve the problems?

7. What are you most proud of in your country?

8. What do you dislike the most about America?

9. Now, there are many Thai students coming to America, you have broken your tradition of living with your parents and I suppose, you will have much more freedom here, so my question is "Do you think this freedom is going to affect your cultural attitude when you go back home?" 
10.What is the economic situation like in Thailand, compared to USA?

11.Do you have any proverbs or values that you believe? Why do you believe in them?

12.Have you heard about the Yandra monk who violated the monk regulations by having intercourse with women? What do you think about him?

The twelfth question appears to be too specific for the subjects. However, this issue is extremely popular in Thailand as the press has published about this news for years; everybody talks about the monk' s violation. The subjects from the pilot study seemed to enjoy responding to this question by sharing their ideas and feelings. This was why this question was chosen.

\section{Preparing the Interview Schedule}

At the beginning, from a list of students who were members of Thai Associated Students at a large university in the western United States, the researcher asked for volunteers as participants in the study. For those interested subjects, consecutive interviews were scheduled to avoid interview subjects communicating the nature of the questions to each other. Each interview was 10 minute long, and the overall process was completed in three and a half hours during the course of one day.

In the next step, I interviewed each subject myself using a stimulated recall technique in Thai. The recorded conversation between the subject and the interviewer was reviewed and the subjects were asked to describe why they had filled/unfilled pauses or retraced/unretraced restarts at points during the conversation. Later, all the audiotaped dialogues were transcribed in order to analyze the subjects' fluency level.

\section{The Process of the Interview}


Each subject was asked to read the consent form and allowed to ask any questions of the researcher. Then the subjects were informed of the purpose of the interview briefly and specifically that it was to obtain the spontaneous responses in L2 by answering both easy and difficult questions. Next, the subject was asked to be inside the room with the interviewer and another person who was responsible for tape recording and time keeping. Although the nature of the interview created a testing-type environment, the interviewer created a friendly atmosphere with the objective of making the subjects feel as though they were having a normal conversation with an American friend.

When the subjects finished their interview, the interviewer gave them their own tapes. They then brought the tapes to the researcher. The researcher played the tapes back to the subjects and stopped the tapes at certain points when hearing the subjects' pauses and restarts. The subjects were asked for the reasons why they produced those behaviors (i.e. filled/unfilled pauses, retraced/unretraced restarts) at certain points. The researcher both recorded and took notes of the responses.

\section{DATA FOR ANALYSIS}

In order to answer these three research questions, both quantitative and qualitative analyses were utilized. The questions were as follows:

1) How many of the intended messages from Thai graduate students in the corpus analyzed are understood by English-native speakers?

2) To what factors do Thai graduate students attribute their fluency related behaviors?

3) How orally fluent are Thai graduate students in the production of English? 
In order to investigate the first research question, both qualitative and quantitative analyses were used. Because the data collected were evaluated by two English native speakers who might have an unequal ability to comprehend non-native messages, analysis of the data in this part was qualitative, even though they are both familiar with non-native speech (one has spent more time teaching English to NNSs than the other and has more experience with international students). The first person who rated the subjects' speech had recently received a TESOL certificate. The second individual had a Master's degree in TESOL and has been an ESL instructor at a university with 14 years teaching experience. Moreover, this individual has experience in evaluating NNSs' speech production by using the Educational Testing Service's SPEAK Test as the criteria. Due to differing amounts of time and professional experience as well as their familiarity with NNSs speech, the second person was considered more likely to comprehend NNSs more often than the first individual.

They were each given the tapes at a different time. They listened to the tapes and evaluated them by scoring each subject. Once they started playing the tapes, they continuously evaluated them until the last one. The possible scores ranged from $0-3$, a format replicated from the ITA Test Score Sheet part III Interactive Language Skills (Smith et al, 1992). The definition of each score was clearly provided to the evaluators (see the whole definition in Appendix A). The definition of each rating of comprehensibility is shown below in Figure 1. 
ITA Test Score Sheet

3 Completely comprehensible. Only a few errors in pronunciation, grammar, fluency or vocabulary.

2 Generally comprehensible. Some errors in pronunciation, grammar, fluency or vocabulary that occasionally interfere with intelligibility.

1 Somewhat comprehensible. Major errors in pronunciation, grammar, fluency and vocabulary that often interfere with intelligibility.

0 Generally incomprehensible. Due to very little ability in pronunciation, grammar, fluency or vocabulary.

Figure 1 from ITA Test Score Sheet by Smith et al, 1992

Tests for Statistical Analyses

Next, the scores rated in this part were analyzed quantitatively to answer the first research question. Cohen's Kappa was used to measure the agreement between two evaluators. Perfect agreement will be a Kappa value at 1 . The first hypothesis indicates that listeners will understand $80 \%$ or less of the subjects' messages due to some errors in pronunciation, grammar, fluency or vocabulary that occasionally interfere with intelligibility. However, due to a negative agreement between two evaluators, Kappa could not be computed. One-tailed t-test was used instead. $100 \%$ was set equal to 3 in the ITA Test Score Sheet. $80 \%$, expected NS comprehension of the subjects' messages, was set equal to 2.4.The null hypothesis was assumed to be true providing that the mean was equal to 2.4 or greater than 2.4. For the research hypothesis, the mean was less than 2.4 . If the results showed that the mean was less than 2.4 , the conclusion was to reject the null hypothesis. 
Analysis of Interview Results

In order to answer the second research question: to what factors do Thai graduate students attribute their fluency related behaviors?, reasons of production of those four features (filled/unfilled pauses and retraced/unretraced restarts) were analyzed according to the results of interviews with the subject. The analysis of the interview results is descriptive and qualitative, without any statistical analysis.

After the interviews with all of the subjects, the researcher discovered varying results. The same answers were grouped under the name of one category. It was usual that one subject contributed various reasons when producing those features which might be similar or completely different to the others' responses. Therefore, one person's answers belonged to more than one category, for example, groping for vocabulary, thinking about questions.

Afterwards, the number of same answers were counted in each category. If the number of the same answers equaled the number of subjects, they were converted to a percentage indicating that 20 subjects with 20 same answers were equivalent to $100 \%$. Then, a graph was used as a means to show the proportion. The descriptive explanation of each category was provided. The graph and explanation will be presented in chapter IV.

Last, quantitative analysis was solely used for the third research question: how orally fluent are Thai graduate students in the production of English? The following six 
sub-questions were selected and the means and standard deviations of the answers were calculated to compare fluent and non-fluent subjects without further statistical tests. T-tests and Man-Whitney U/Wilcoxon Rank Sum W Test were used to strengthen the validity of the third question in the study.

The six sub-questions follow. Their definitions were indicated on p. 4, Chapter I.

1) How many filled pauses per 100 semantic words do the subjects produce within the utterances?

2) How many unfilled pauses per 100 semantic words do the subjects produce within the utterances?

3) How many times do retraced restarts: repetition and insertion per 100 semantic words occur?

4) How many times do unretraced restarts per 100 semantic words occur?

5) How many semantic words per minute (WPM)--words that contain semantic information--excluding four features (filled/unfilled pauses and retraced/unretraced restarts) do the subjects produce?

6) How many total words per minute (WPM) do the subjects produce?

\section{Eluency Measures}

Speech production from the interviews was examined by using six fluency/nonfluency markers such as hesitation phenomena (filled/unfilled pauses), repair (retraced/unretraced restarts) and rate of speech (total number of words per minute/total number of semantic units per minute) as the criteria to determine their fluency level. 
Fluency was defined in chapter II as speaking with ease--a fluent speaker does not need to spend much thought or energy on L2 production, but rather produces words that are strung together within utterances with nonfunctional pauses in a smooth fashion (Starkweather, 1987; Gelderen, 1991; Riggenbach, 1991). Moreover, Schmidt (1992) says that "non-fluent speech is effortful and requires a great deal of attention, so that nonfluent speakers exhibit many hesitations and other manifestations of groping words and attempting to combine them into utterances" (p.358). These definitions were used as parameters in order to evaluate the subjects' fluency in L2. The definitions of these features (replicated from Riggenbach, 1991) are described below within the analyses procedures.

Audiotaped dialogues were transcribed and the 5 longest discourse passages were selected. The reasons for not selecting everything were 1) some subjects' responses were too short to be analyzed and 2) some responses were occasionally interrupted by the interviewer.

\section{Analysis of Total WPM}

WPM - the count for all words including partial words (using the criterion that partial words contain not just an initial consonant but also a vowel and thus are recognizable as words)

It was found that it was less time-consuming to analyze question 6: how many total WPM do the subjects produce, after that question 5, and then to go back to question 1 through 4 , in numerical order. The 5 longest passages were timed. Every word in each passage was counted. The time for responses varied. For example, subject no. 1 produced 
39 words within 29 seconds for the first excerpt, and 79 words within 38 seconds for the second excerpt. Twenty-nine and 38 seconds were converted to minutes by dividing by 60. The time from 5 passages was added. Then, every word from 5 passages was added. After that, the total words were multiplied by one and divided by the time added. An excerpt from the transcript of Tlexemplifies T1's production of 39 words within 29 seconds.

T1: um. how to explain I'm not good at English uh-hu (.) so it cause many failure in the how to say in the government system how to say (.) and (.) they have a consequently (.) how to say (laugh) (.) effect many many thing

\section{Analysis of Semantic WPM}

Semantic WPM - the count for words that contain semantic information excluding filled/unfilled pauses and retraced/unretraced restarts.

How many semantic WPM do the subjects produce? Semantic WPM did not include filled/unfilled pauses, retraced: repetition and insertion and unretraced restarts (see the definitions in p. 4, Chapter I). Besides the rate of speech production, the amount of information conveyed was another main feature used to judge the fluency level of the subjects. Procedures of the counting are found in Appendix B and their reasons are discussed in validity and reliability of data analysis. The same 5 discourse passages were used. The semantic words were counted. The same method used to answer the question 6 was used. The times converted to minutes from 5 passages were added. Every semantic word each passage was added. Next, the total semantic words were multiplied by one and divided by the time added. The same excerpt from the transcript of T1 given above exemplifies Tl's production of 36 semantic words within 29 seconds. 
T1: um. how to explain I'm not good at English [uh-hu] (.) so it cause many failure [in the] how to say [in the] government system how to say (.) and (.) they have a consequently (.) how to say (laugh) (.) effect [many many] thing

Words in the brackets were not counted as semantic words because they were filled pauses and retraced restarts.

\section{Analysis of Filled Pauses}

Filled pause - voiced fillers, which do not normally contribute additional lexical information. There are two kinds of filled pause in the study.

a) nonlexical - ("um," "uh",) fillers that are not recognized as semantic words because they contain little or no semantic information

b) lexical - (y'know," "I mean") fillers that are recognized as words but in context contribute little or no semantic information

The first sub-question was: how many filled pauses out of 100 semantic words did the subject produce within the utterances? The semantic words counted in each passage were used as a core. Filled pauses were counted in each passage. Then, those filled pauses were added. In order to convert them to a percentage, the total filled pauses were multiplied by 100 and divided by the semantic words added.

Lexical fillers were inconsistent sometimes. Therefore, the procedures of counting them were defined and shown in Appendix B. For example, "like a" was counted as a lexical filler, while "like" which means "for example" was not counted as a filled pause, but a semantic word. Also, filled pauses that occurred at the beginning of the utterances were not counted. For instance, "Ah-ha" that occurred when T16 started his utterances was not counted. An example from subject T16 shows the way lexical fillers were counted. They are underlined below. 
T16: Ah-ha because firstly I choose the, to study in USA because when I watch movie like a when I watch American movie and British movie like a the Bri-the British movie hard to understand like a they accent something like that but American is more easier to to understand and it's like a fashion I don't know something like a fashion American country like that when, when first when the people graduate from the university for their own bachelor and they try to (.) go to America for the Master's degree something like that yeah (.) and I don't know because American, America is like a lot of nation so they have like a progress in a technology and everything ah-ha and why I choose Oregon firstly when I apply I, I got the I-twenty from Texas and I got I-twenty from Oregon State I mean secondly but I choose Oregon because I, I, I don't like the weather in Texas because like a hot weather and my, my friend told me there is a snow in Oregon also so I choose to study Oregon, I want to see the snow.

In addition, lexical fillers (i.e. "you know I mean", "or something", or "something like that", "like a", "like that" etc) were counted only as one filled pause because they are one lexical phrase. The reason for considering these lexical fillers was because they provided no semantic information to the listeners. Instead, they functioned as an interactional device between speakers and listeners during communication. Riggenbach (1991, P.426) states that:

As Shiffrin (1987) and Fiksdal (1990) point out, these might more accurately be considered "discourse markers" rather than lexical filled pauses, because they most definitely perform important interactional functions. However, this discourse function is not necessarily related to literal meaning, and thus in many contexts the contribution of such fillers is not primarily semantic but interactional. In this study, which focuses on nonnative speaker fluency, characterizing these markers as one type of filler revealed some interesting differences between fluent and nonfluent usage.

\section{Analysis of Unfilled Pauses}

Unfilled pause - a silence of 1 second or greater indicated by a period within parentheses (.). 
How many unfilled pauses out of 100 semantic words do the subjects produce within utterances? In this study, the researcher was not capable of detecting unfilled pauses that were less than one second due to lack of a special stopwatch. Unfilled pauses that were marked as non-fluency behavior in this study involved a silence of one second or greater, indicated by a period within parentheses (.) (see speech samples in Appendix C.).

Similarly to the analysis of filled pauses, the same 5 discourse passages were used. Unfilled pauses were counted. The total unfilled pauses were multiplied by 100 and divided by the semantic words added.

\section{Analysis of Retraced restarts}

Repair phenomena

retraced restart - reformulations in which part of the original utterance is repeated repetition - exact adjacent repeats of sounds, syllables, words, or phrases insertion - a retraced restart in which new (unretraced lexical items are added)

How many times do retraced restarts out of 100 semantic words occur? The same

5 excerpts were used. The frequencies of the occurrences of retraced restarts were counted. If repetitions and insertions happened twice, they were counted as one retraced restart (see in Appendix B.) An excerpt from the transcript of T7 exemplifies this.

T7: Uh-huh. ah. the first surprised, I think is the environment (.) it have a lot of building the the place is very kind of person ah. I, I, I see a lot of people from many national nationality uh-huh and yeah and like a he greeting or welcoming me when I, when I see a people in street he always say hi, or that thing.

"the the" was counted as one restart and "I, I, I" as twice. 
Then, those retraced restarts were added. In order to convert them to a percentage, the total retraced restarts were multiplied by 100 and divided by the semantic words added.

\section{Analysis of Unretraced Restarts}

unretraced restart - reformulations in which the original utterance is rejected (false start) How much does a person reject the original utterance? (more than one word as opposed to only one word)

How many times do unretraced restarts per 100 semantic words occur? Unretraced restarts were counted from the same 5 excerpts. Unretraced restarts were added. The total of unretraced restarts were multiplied by 100 and divided by semantic words added.

Rhode (1985) infers that there is a higher tendency for more fluent speakers to reject the original utterance quicker than the less fluent speakers. Nevertheless, in this study, unretraced restarts (either more than one word or only one word) were both counted as one occurrence of unretraced restarts because Riggenbach (1991) has suggested that if at least three features occurred together as a chunk of disfluencies within an utterance, they can be considered non-fluency behaviors. Therefore, those unretraced restarts followed by the other non-fluency features were counted in the study even though the subjects rejected the original utterances of only one word. An example of a chunk of disfluencies is shown in the following excerpt:

T1: uh-huh. the festival in Thailand I think it relate to the religion circumstance (.) so when we celebrate these day, this day we have to er. event er. the religion events and (.) just er. and ot-another just party like look like that but here there are on-er most of the (.) tradi-festival here I, I, I don't know, I don't 
know if they going to church or not but mostly they just have ah. enjoying they themselves

According to the example, beginning from the unfilled pauses, the features that follow are an insertion, a filled pause, an insertion, an unfilled pause, an unretraced restart, an insertion, a filled pause, an unfilled pause, an unretraced restart, two repetitions, a repetition, a filled pause and an retrace restart. Obviously, unretraced restarts (only one word) needed to be counted. Riggenbach (1991, p. 427) says that "The consideration of the environment of a feature is important and justifiable because an isolated pause may be considered forgivable, whereas a pause that occurs with, say, a repetition and an unfilled pause may not be."

After the analyses of every fluency-related feature, the raw frequency data were organized into Table IV, p. 59. Summaries of means and standard deviations of filled/unfilled pauses, retraced/unretraced restarts and total WPM/semantic WPM of 18 non-fluent and 2 fluent subjects were used to answer the third question regarding the subjects' oral fluency. The notion of fluency and its elements were used as cut-off points in judging the subjects' L2 production. There were only two subjects that could be characterized as fluent speakers. T4 produced 170 WPM and 144.6154 semantic WPM and T19, 158.1818 WPM and 146.9697 semantic WPM respectively. Moreover, out of 100 semantic words, $\mathrm{T} 4$ had only $8.80 \%$ of filled pauses, $0.27 \%$ of unfilled pauses and $2.13 \%$ of retraced restarts and $0.27 \%$ of unretraced restarts. Totally, there were $11.47 \%$ of four non-fluency features occurring out of 100 semantic words. Similarly, T19 had $2.06 \%$ of filled pauses, $0.62 \%$ of unfilled pauses, $3.71 \%$ of retraced restarts, and $0.62 \%$ of 
unretraced restarts out of 100 semantic words. T19, thus, produced $7.01 \%$ of four features out of 100 semantic words. According to the raw data, English spoken by T4 and T19 appeared to be more in line with the notion of fluency by many researchers (Arevart \& Nation, 1991; Gelderen, 1994; Lennon, 1990; Rehbein, 1987; Riggenbach, 1991; Schmidt, 1992; and Starkweather, 1987) because they both produced smooth utterances along with a relatively high rate of speech and a relatively low pause count.

\section{Tests for Statistical Analyses}

Quantitative analyses of hesitation, repair phenomena and rate of speech were supplemently examined using t-tests and the Mann-Whitney U/Wilcoxon Rank Sum W Test. Comparison between two groups, fast and slow, by using WPM and semantic WPM as criteria in breaking the group of the subjects was a means to find out significant differences between the two groups. The cut-off points were selected by the mean of total WPM and semantic WPM the subjects produced. If the subjects produced less than 120 WPM and less than 99 semantic WPM, they were considered to be in the slow group. In contrast, if they produced at 120 WPM or greater and at 99 semantic WPM or greater, they were in the fast group. Consequently, there were 11 subjects in the slow group and 9 subjects in the fast group. The reason for dividing the subjects into two groups was to produce two distinct groups, for comparison and statistical testing.

The t-test and the Mann-Whitney U/ Wilcoxon Rank Sum W Test were appropriate to this study. T-test is a parametric test and it is suitable for two reasons. First, this study was investigating the differences of the mean between two populations. 
Second, there were twenty subjects in the study, considered a small sample. This study had two independent volunteered samples and then two sample t-tests were used. Two sample t-test are only valid if the two populations are very similar to each other; they have to be normally distributed and have to have equal or close to equal variances. According to Levene's Test for Equality of Variances, if the $p$ value is smaller than .05 , the evidence shows that unequal variances will be considered as results. In contrast, if the $\mathrm{p}$ value is above .05 , equal variances will be considered instead. Also, Mann-Whitney U/ Wilcoxon Rank Sum W Test was used as a back up of the t-test to make certain of the results and ensure accuracy. If these two statistical tests support each other, statistically significant differences will prove comparable. Mann-Whitney U/ Wilcoxon Rank Sum W Test is a non-parametric test which does not assume normal distributions or equal variances. Alpha level for statistical decision was set at $\mathrm{p}<.05$ and in all the tables in this study, statistically significant differences were marked with an asterisk.

In conclusion, the means and the standard deviations of a number of occurrences of each variable: filled/unfilled pauses, retraced/unretraced restarts and the sum of four features of two groups: fluent and non-fluent were presented to answer the third question from the raw frequencies of those features without statistical analysis due to the inequality of the number of two groups of the subjects.

\section{Validity and Reliability of the Data Analysis}

Riggenbach (1991) found in her study that results using this kind of data can be "tentative and are limited to particular subjects" (p.425). The researcher of this study 
found the same to be true. Riggenbach's research is descriptive and exploratory. Although the results are of a speculative nature, and not therefore generalizable to other NNSs, they may nevertheless contribute to establishing descriptions of fluency.

The purpose of this study is to provide more understanding of the phenomenon of fluency and its features. If people have a greater understanding of what fluency is comprised of, they will be more able to distinguish between fluency and non-fluency which can affect the teaching and testing of languages, as well as research on second language acquisition processes. Furthermore, investigation into fluency would become more reliable.

In order to establish the reliability of the data analysis of this study, interrelater reliability was used. Also, clear definitions of filled/unfilled pauses, retraced/unretraced restarts and total WPM and semantic WPM were provided and counted consistently when analyzing the transcript of the subjects' speech.

For the first research question regarding the number of intended messages understood, an interrater reliability evaluation was conducted. This evaluation was carried out by a senior instructor of the ESL program at the university. She is familiar with using SPEAK Test to evaluate NNSs' speech as indicated earlier in the NS participants section.

For the second question regarding students attribute their behaviors to hesitation and repairs, retrospection was the method used to establish more reliability . The data were collected immediately after the subjects had finished their interviews with the NS. 
The subjects were asked to retrospect by using a stimulated recall technique. They listened to the recorded interview tapes and answered the second question. This retrospection could decrease the researchers' biases and assumptions about the responses.

For the third research question regarding oral fluency, because the researcher needed an individual who was familiar with spoken English with a Thai accent, and was capable of understanding the words that the subjects said, a Thai student was asked to transcribe about 30 minutes of the tapes. She had just finished her Master's degree in Educational Policy Foundation and Administration. Those tapes were selected randomly. Because of the time consumed in transcribing, she spent about one and a half hours transcribing one tape of subject T6. We discussed earlier that everything she heard would be transcribed. At completion, she had 800 words of the whole tape, whereas, I counted 808. Then, we circled the words that were not the same or were missing or words that were added which another did not have. We listened to that tape together and finally were able to reach the same agreement that most of the time she missed nonlexical fillers like "ah", "uh", "um", as well as articles like "the", prepositions like "to". Also, the same word was heard but transcribed as different words, for example, "fifteen - sixteen" and "I'm - and".

There was a big concern about counting those four related fluency features; therefore procedures of counting are described in detail in Appendix B, and sometimes the same words were interpreted as different functions depending on their occurrences in a different environment. For instance, "like a" was counted as a filled pause (lexical 
fillers), while "like" which means "for example" was counted as a semantic word. T16 is a good example of this (shown earlier). Another example was "It's like a tradition" and "It's like when". The former was counted as two semantic words whereas the latter was considered as one filled pause. All of these are shown in Appendix B. Moreover, procedures of counting words were included. For instance, "cannot", "I-twenty", and "GPA" were counted as one word and one semantic word. The repetitions of the first syllable like "be-because", "fam-family", "Bri-British"--be, fam, Bri-- were not counted as semantic words. The researcher attempted to keep procedures of counting consistent as much as possible throughout the data analyses.

\section{SUMMARY}

This study's objectives were to answer three research questions and to test three hypotheses:

1) How many of the intended messages in the corpus analyzed from Thai graduate students are understood by English native speakers?

2) To what factors do Thai graduate students attribute their fluency-related behaviors?

3) How orally fluent are Thai graduate students in the production of English?

To answer question 1, inter-rater reliability was first examined and one t-test was conducted by indicating that the mean was equal to 2.4 . For question 2 , data analysis was descriptive and qualitative. Last, this study examined the high-low occurrences of features that contribute to the characterization of fluent versus non-fluent subjects determined by the differences of the means and standard deviations of the two groups. 
Hesitation (filled/unfilled pauses), repairs (retraced/unretraced restarts) and rate of speech (total WPM/semantic WPM) were investigated and differences of six variables produced by the subjects between two groups (fast versus slow) speaking English were compared by using t-test and Mann-Whitney U/Wilcoxon Rank Sum W Test to increase the validity of the third question in the study. Chapter IV presents results with respect to the three research questions. 


\section{CHAPTER IV}

\section{RESULTS AND DISCUSSION}

Results of this study are presented and discussed in this chapter. First, the study reveals the amount of the listeners' understanding towards the subjects' messages. Second, it shows the interview results from the speakers themselves regarding their nonfluent behaviors (hesitation and repair) occurring during the conversation analyzed qualitatively by means of content analysis. Moreover, results of the analysis of subjects' actual speech production are reported. Six related fluency features (filled/unfilled pauses, retraced/unretraced restarts and total WPM and semantic WPM) are investigated both with and without statistical analysis. Transcription samples of the speech data are shown in Appendix $\mathrm{C}$, and raw frequencies of the occurrences of those features used to answer question 3 are found in Table IV.

To analyze the first research question regarding the number of intended messages understood, Cohen's Kappa and t-tests were used. For the second question, interview results were illustrated by a graph and explained. For the third research question, means and standard deviations of a number of occurrences of hesitation and repair as well as of rate of speech between fluent and non-fluent subjects were compared. Fluency level of the subjects was judged by the criteria defined in chapter II. To strengthen the validity of the analysis of the third question in this study, the t-test and the Mann Whitney 
U/Wilcoxon Rank Sum W Test were used to compare by dividing the subjects into two groups based on their rate of speech. Total WPM and semantic WPM at 120 and 99 or greater were the cut-off point between the fast and slow group. The cut-off points were calculated from the means of all of the subjects' rate of speech (total WPM and semantic WPM). To validate the study and utilize statistical tests, the researcher divided the two groups into equal numbers. A criterion level for statistical decisions was set at $\mathrm{P}<.05$ and statistically significant differences are marked with an asterisk. Raw frequency of features that have been ascribed to fluency (hesitation: filled/unfilled pauses, repair: unretraced/retraced restarts, and rate of speech: total WPM and semantic WPM are included in the following tables and in Appendix D.

\section{The First Research Question/Hypothesis}

\section{How Many of the Intended Messages in the Corpus Analyzed From Thai Graduate Students are Understood by Native English Speakers?}

Hypothesis 1: Listeners will understand approximately $80 \%$ or less of the subjects' messages due to errors in pronunciation, grammar, fluency, or vocabulary that occasionally interfere with intelligibility.

Cohen's Kappa was used to measure the agreement between two evaluators. The results showed that there was a disagreement between two evaluators because the Kappa evaluation value of -.018 was not perfect. Perfect agreement would be a Kappa value of 1. The result was much closer to 0 rather than 1 . This indicated that the two raters were fairly different in their agreements. One rater is an ESL instructor with a TESOL 
certificate and the other is an ESL instructor with a Master's degree in TESOL. Then, one-tailed t-test was conducted in order to find out statistically significant differences in the mean of intended messages understood. The mean was calculated as $80 \%$ which was equivalent to 2.4 in the ITA Test Score Sheet. The mean was set equal to 2.4 and 2.4 is equal to $80 \%$. The null hypothesis was first assumed to be true that the raters comprehended the subjects' L2 production 2.4 or greater, while the research hypothesis was in contrast to the null hypothesis. Table II shows the result of the first evaluator scores.

TABLE II

The First Evaluator Scores

One-tailed t-test

\begin{tabular}{lrrrr} 
Variable & Number of Cases & Mean & SD & SE of Mean \\
\hline The first evaluator & 20 & 1.6000 & .771 & .172
\end{tabular}

Test Value $=2.4$

\begin{tabular}{lll|lll} 
Mean & $95 \%$ CI & & & \\
Difference & Lower & Upper & t-value & df & 1 -tail sig \\
\hline-.80 & -.1 .161 & -.439 & -.4 .64 & 19 & p $>* .00$
\end{tabular}

The mean calculated from the first evaluator was 1.6. This supported the first research hypothesis because the mean was below 2.4 . In addition, a highly significant difference was found because $\mathrm{p}$ value for the test is less than * .00 . Table III shows the results of the evaluation of the second evaluator.

TABLE III

The Second Evaluator Scores 
One-tailed t-test

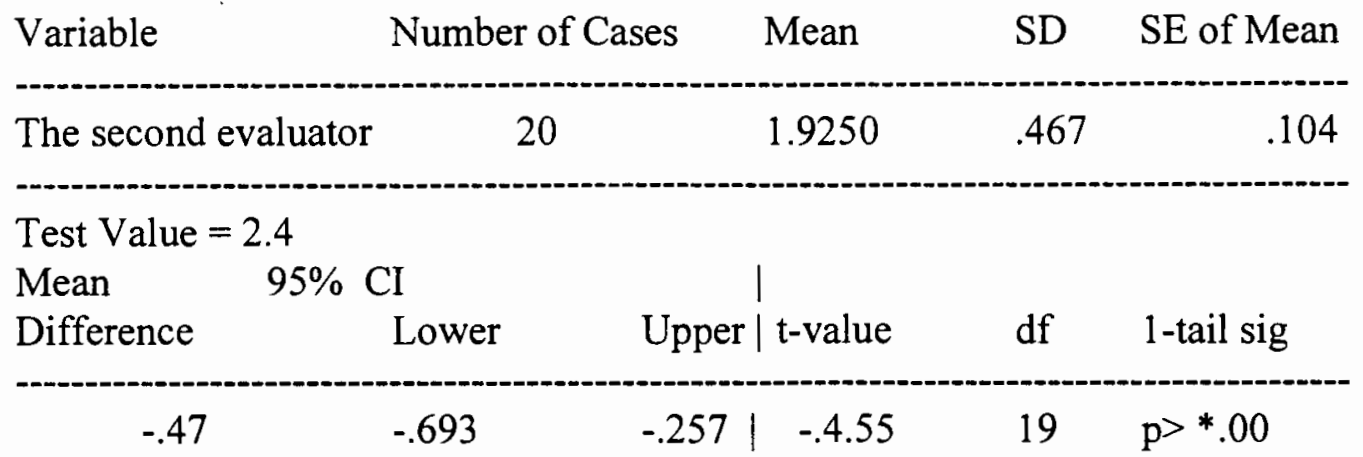

The mean of Table III was 1.925 which was lower than 2.4 . It is statistically significant difference because the $\mathrm{p}$ value is less than *.00. The result supported the first research hypothesis in that Listeners did understand less than $80 \%$ of the subjects' messages due to errors in pronunciation, grammar, fluency, or vocabulary that occasionally interfered with intelligibility.

However, due to the disagreement between two raters, the second rater's results were chosen to answer the first research question because the individual has more professional experience in evaluating NNSs speech production especially for International Teaching Assistants using the SPEAK Test (1982). It was concluded that strong evidence was found to support the first hypothesis and to reject the null hypothesis.

The Second Research Question/Hypothesis

To What Factors do Thai Graduate Students Attribute Their Fluency-Related Behaviors?

Hypothesis 2 : Speakers' encoding capacity particularly in lexis significantly influences the fluency level of their spoken English. 
The interview results revealed Thai graduate students' problems in L2 production. The results are presented in the Table IV following. The explanations are provided. Groping For Vocabulary

All of the subjects reported problems in vocabulary. They said they produced hesitation and repairs because they did not know the vocabulary, particularly words being used in daily life. This implies that they might have less social interaction with speakers of the target language, resulting in limited vocabulary. Also, they said they could not think of the vocabulary immediately even though they knew it. They said they needed more time to come up with it and often the vocabulary they wanted arose just after the conversation. Moreover, some Thai words and phrases are culture specific and seldom used by English NSs (see the example below). The subjects searched for words and decided which exact lexical item would used during speech execution. Some demonstrated circumlocution or paraphrasing to continue expressing their intended messages as well as to keep the conversation flowing. Other subjects demonstrated filled/unfilled pauses instead. The following excerpts demonstrate this groping for vocabulary:

T2: um. (.) we we usually go to the temple to (.) I don't know what to how to call it in English

I: do the merit?

T2: I don't know yeah do merit, I I don't know 
Figure 2 Interview Results Chart

\begin{tabular}{|r|l|r|} 
Subjects & \multicolumn{1}{|c|}{ Reasons based on 20 subjects } & Percentage \\
\hline 20 & Groping for vocabulary & 100 \\
\hline 14 & Attempting to express the missing intended message & 70 \\
\hline 8 & Thinking about questions & 40 \\
\hline 6 & Monitoring the speech production & 35 \\
\hline 4 & Attempting to combine words into utterances & 20 \\
\hline 4 & Translating from $L 1$ to $L 2$ & 20 \\
\hline 2 & Attempting to clarify the ideas & 10 \\
\hline 1 & Worrying about pronunciation & 5 \\
\hline
\end{tabular}

Interview Results Chart

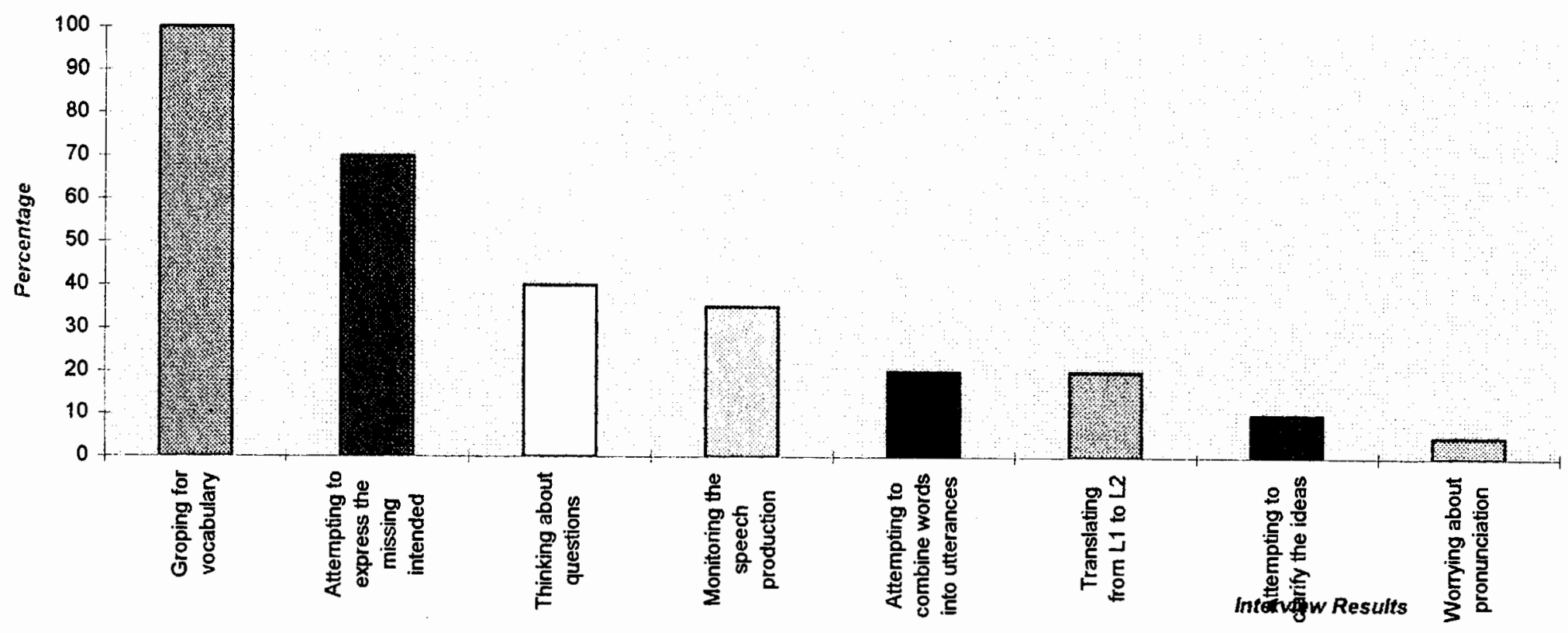


T2: um. Actually I ap I apply to study in (.) Australia and (.) um. England too and I I didn't give up I mean I mean I didn't keep in touch with them and I apply to study in five universities in United States I mean but [the name of the university] accepted me (laugh)

T5: (.) um. (.) ah. the the New Year of the Thailand that uh. (.) call Songkran they uh. (.) in the summer so we (.) uh. like a put the water in the body to everyone (.) that uh. make me people in Thailand colder because the hot water uh. the hot weather in Thailand very hot.

T6: I hate it, I hate that that monk because because it's the worst case of the of the breaking the rule rule for the monk and (.) he did it (.) like (.) many times he did it (.) without any any (.) any fear or any I don't know how to say any uh. (.) I don't know what to say.

I: o.k. I know what you mean he did it without thinking about the consequences like he can do whatever he wants?

T6: No when you do something bad you have like

I: oh! a conscience

T10: Oh. well question (laugh) (.) how to say the people pen rabieb nia pasa unglish waa ngai [a Thai sentence] um. how to say like the people (.) very I, I can't think of the word in English but it's like when, when you buy, go to buy food or something you kind of stand on the line and uh. it's kind of (.) you have a lot of (.) things done for handicap, that's very good.

T17: Yeah (.) ah. well something people know um. (.) in the government some ah. (.) Minister, I I [murmuring a sentence] yeah like uh. they may ah. suppose there's a construction um. and well so many company still come to um. I don't know the word that I think you understand so um. who who give the high high price something like that

I: $\quad$ give a bid

Attempting to Express the Missing Intended Message

The subjects reported that because of language barrier, they could not express themselves as clearly as they wanted. Almost every time, there were some messages missing. They paused in order to express what they actually meant to say but couldn't, so they just ignored those messages. Quite often, the interviewer attempted to guess from 
their nonverbal communication or words and asked them whether or not these were their intended messages. They would answer only yes or no questions. If those messages were still not the intended ones, they would say something more until the interviewer could comprehend them.

Thinking about Questions

The Thai subjects said questions were not difficult but they never answered these types of questions before so they had to think. Once they started their sentences, they felt that it was not what they wanted to say. They did not feel that they were actually answering the questions. All said that if it was in Thai, they would do better than this. Some revealed that they had never thought about the question in English before, so it was difficult for them to simultaneously plan utterances and produce them easily and effectively.

Monitoring Their Own Speech Production

Their concerns were mostly about two issues: the correction of grammatical forms and the correction of information. First, their repeats occurred because the subjects worried about grammatical correctness, particularly tense. They said when they found errors or inappropriateness in their production, they had to correct them first and then continue the intended messages. They could not ignore those errors. The following examples from the data illustrate correction of both grammatical forms and information by $\mathrm{T} 10$ and $\mathrm{T} 17$.

T10: (.) what do I think about those, hum. I (.) actually I I don't like it very much because I think that um. (.) people will um. (.) respect more to less have less res, respect to Buddha um. Buddhism so that uh. actually the idea for Bud, 
Buddhism is to (.) um. how to say, to be to teach people to be a good person or whatever but because people always just look at the peop and look at the monk or something and then just you like orr [Thai proclamation] if they are good, so that they can teach us if they, if they are not good, they couldn't actually I mean if we just feel that they know something and they teach us to about the concept and we just accept only the concept that's good but because people are more like look at the people, how to say, just stick to the person to person so

T17: um. oh. yeah. maybe (.) because I (.) well like we, I've seen many of American movies and um. I been here once I mean, I had (.) well, I don't know what to say I mean I was here once before, I had been here once before I I uh. I came here this time and um. my sister used to study here three years ago something like that

After listening to the tape, T12 found a grammatical error that he had not detected during his speech. He was so surprised and ashamed that he had ignored this error without correcting it. From the conversation, T16. said "oh! New York it's a lot much more bigger than Bangkok." This example showed the subject's concern with the correction of grammatical forms.

T17's excerpt supports Krashen's study (1981) that over self-monitoring leads to interference with the speech production flow and causes non-fluent speech. He calls this behavior "monitor overuse" referring to people who perform too much editing on their L2 output. If the speakers show overconcern with correctness of linguistic form, they, then, are unable to speak with fluency (Krashen, 1981). On the other hand, monitor underusers may or may not be capable of detecting the errors that they are making; they often do not demonstrate an awareness of their linguistic knowledge of the sentences they speak. Some fluent NNSs are still rated as non-fluent in a number of studies because of their ungrammaticality. Optimal users tend to know when they should self-correct and such 
corrections will not get in the way of communication (Krashen, 1981). As Beebe (1988) mentions, the learner says, "I know that I have produced an error, and I may know the correct form, but I'd rather not bother editing my output because I know that it will not interfere with what I have to say" (p.37). Second, Beebe (1988) explains that a good language learner should have an intention to take chances of being a risk taker to attempt to get his/her intended messages across.

Attempting to Combine Words into Utterances

The subjects reported that they had syntactical problems in constructing sentences: particularly when the sentences were more complex, they were more likely to pause. When telling the stories, they felt like they could not combine words into comprehensible sentences or convey their ideas using proper tone inflections. It was not the same when telling stories in their native language. Consequently, they parsed words or phrases and assumed that NSs would be able to guess and understand the carried messages. Also, they used nonverbal communication to assist the interlocutor in better understanding.

Translating Thai to English During the Speech

The subjects corrected their sentences because they found that the syntax of the utterances sounded Thai rather than English but often they preferred to ignore it. Their concern was vocabulary. They said they knew the vocabulary they wanted to use but they felt that those words did not have equal meanings to the words in Thai they wanted to express. For example,

T5: Most proud (.) I proud of (.) tradition in Thailand, I don't know what you call 
like a (.) um. (.) Ram Thai [Thai word] I don't know what you call.

Subject T5 reported that she knew that she could use the word "dance" or "performance" but she did not use either because both words provided slightly different meanings from what she wanted. Those words were too rigid and made Thai words lose their beauty and authenticity. After making an effort at finding words that had the same meaning in Thai, and finally had to give up. The subjects said they were frustrated when this event happened. Expressing Thai words made a lot of sense to them and had meaning in depth but there were no such words that were exactly equal in terms of meaning in English. T3 said that he translated a Thai sentence into English even though he realized that it was a strange sentence. He hoped that if he stayed in the United States longer, he would someday be able to think in English during speech production.

T3: Most proud (.) (ps) I think uh. (.) Thai ah. (.) the word Thai means we (ps) (.) independent we (.) uh. we have never been (.) uh. under (.) under rule for example India uh. English England take charge tor [slip a Thai word] for many year and use they change uh. their official language to be the English one but Thailand even though we (.) we (.) no have ah. some kind like that o.k. uh. some some people say o.k. for the civilized, civilization country when wey when they came came to take over that (.) uh. develop country they bring the technology bring the knowledge but uh. in my opinion even though we do not have that kind of uh. new technology but we very proud that we (.) independent not ah. (.) under er. (.) under some countries some France, Portuguese and English England and we have uh. we have the (.) kings that ah. we call Rama the Fifth he was very clever when French and England (.) k-come to our country maybe they can claim that uh. we not ah. civilize country we have uh. good (.) study (.) system we not have some civilize thing that they have so they try to take in charge but uh. but uh. (.) that king sent his son, sent sometime sent his daughter to go oversea to study and bring the technology and knowledge to develop. 
In Thai, the verb "bring" does not need an indirect object while in English "bring" does need both direct and indirect objects such as "bring Thailand the new technology and knowledge."

\section{Attempting to Clarify Ideas}

Due to cultural differences between, Thailand and the United States, two subjects pointed out that they paused because they were finding ways to explain to the NS so that NS would be able to visualize their explanation.

\section{Worrying about Pronunciation}

There was one person who was concerned about pronunciation. He reported he repeated words because he stressed them at the wrong syllable and noticed the interviewer's facial expression that she did not understand. He decided to say that word again.

\section{SUMMARY}

Interview data reinforced the existence of non-fluency behavior in much of the subject production data. Subjects were aware of their lack of vocabulary as the main problem. Also, they were concerned that their conscious thinking process, which is in The Thai language, was properly translated into the target language with a degree of accuracy in sentence structures. Therefore hypothesis 2 was supported because all of the subjects reported in consensus that their major problem was vocabulary. They did not know enough vocabulary to express their intended messages effectively and clearly. This interrupted the smoothness and continuity of speech. As Fillmore (1979) explains, if a 
speaker is not syntactically fluent, semantically fluent, pragmatically fluent, he/she is likely to be less fluent in his/her speech production than fluent speakers. In addition, speakers who have less vocabulary will have problems knowing what to say under a wide variety of social circumstances.

The Third Research Question/ Hypothesis

\section{How Orally Fluent are Thai Graduate Students in the Production of English?}

Hypothesis 3: English-speaking Thai graduate students in this study are unable to simultaneously plan utterances and produce them in a smooth manner.

Frequencies of filled/unfilled pauses and retraced/unretraced restarts as well as WPM and semantic WPM are presented in Table IV. Also the means and standard deviations of these frequencies were calculated to examine the third question. 
TABLE IV

Frequencies of the Number of Fluency-Related Features

\begin{tabular}{|c|c|c|c|c|c|c|c|}
\hline ubject & filled & unfilled & retrace & un-re & sum & $\longdiv { w p m }$ & se-wpm \\
\hline 1 & $7.87 \%$ & $6.94 \%$ & $6.02 \%$ & $4.63 \%$ & $25.46 \%$ & 99.62963 & 79.62963 \\
\hline 2 & $\mid 7.98 \%$ & $7.45 \%$ & $13.83 \%$ & $2.13 \%$ & $31.38 \%$ & 100.5405 & 76.21622 \\
\hline 3 & $8.91 \%$ & $4.82 \%$ & $4.23 \%$ & $0.73 \%$ & $18.69 \%$ & 121.6923 & 105.3846 \\
\hline 4 & $8.80 \%$ & $0.27 \%$ & $2.13 \%$ & $0.27 \%$ & $11.47 \%$ & 170 & 144.6154 \\
\hline 5 & $11.69 \%$ & $12.12 \%$ & $3.03 \%$ & $0.87 \%$ & $27.71 \%$ & 73.86555 & 58.23529 \\
\hline 6 & $5.11 \%$ & $8.71 \%$ & $8.11 \%$ & $0.90 \%$ & $22.82 \%$ & 106.9912 & 88.40708 \\
\hline 7 & $6.47 \%$ & $0.43 \%$ & $9.48 \%$ & $0.86 \%$ & $17.24 \%$ & 136.4885 & 106.2595 \\
\hline 8 & $4.14 \%$ & $8.28 \%$ & $3.45 \%$ & $1.38 \%$ & $17.24 \%$ & 97.14286 & 82.85714 \\
\hline 9 & $11.76 \%$ & $5.88 \%$ & $8.40 \%$ & $1.68 \%$ & $27.73 \%$ & 112.7711 & 86.0241 \\
\hline 10 & $9.12 \%$ & $3.22 \%$ & $4.29 \%$ & $1.34 \%$ & $17.96 \%$ & 120.5455 & 101.7273 \\
\hline 11 & $5.15 \%$ & $1.90 \%$ & $10.30 \%$ & $2.17 \%$ & $19.51 \%$ & 122.1525 & 99.28251 \\
\hline 12 & $6.67 \%$ & $4.24 \%$ & $10.91 \%$ & $0 \%$ & $22 \%$ & 111.8182 & $\overline{9 c}$ \\
\hline 13 & $8.78 \%$ & $4.20 \%$ & $15.65 \%$ & $1.91 \%$ & $30.53 \%$ & 114.2857 & 83.1746 \\
\hline 14 & $8.01 \%$ & $3.52 \%$ & $2.54 \%$ & $1.37 \%$ & $15.43 \%$ & 129.7872 & 108.9362 \\
\hline 15 & $5.05 \%$ & $3.37 \%$ & $5.72 \%$ & $1.01 \%$ & $15.15 \%$ & 131.625 & 111.375 \\
\hline 16 & $8.87 \%$ & $2.56 \%$ & $8.36 \%$ & $1.54 \%$ & $21.33 \%$ & 158.1818 & 122.9371 \\
\hline 17) & $24.21 \%$ & $3.97 \%$ & $9.52 \%$ & $1.59 \%$ & $39.29 \%$ & 117.6923 & 83.0769 \\
\hline 18 & $9.09 \%$ & $3.03 \%$ & $5.68 \%$ & $1.36 \%$ & $19.17 \%$ & 99.48187 & 82.075 \\
\hline 19 & $2.06 \%$ & $0.62 \%$ & $3.71 \%$ & $0.62 \%$ & $7.01 \%$ & 158.1818 & 146.969 \\
\hline 20 & $12.11 \%$ & $1.86 \%$ & $9.94 \%$ & $0.31 \%$ & $24.22 \%$ & 114.3925 & 90.2803 \\
\hline
\end{tabular}

As can be seen from Table IV, there were two subjects considered to be fluent speakers, T4 and T19 because they both fit well in the parameter of fluency, they spoke English quickly with few pauses and restarts. In contrast, the 18 subjects produced a higher volume of filled/unfilled pauses and retraced/unretraced restarts than those 2 speakers. Additionally, they produced a lower volume of WPM and semantic WPM than those 2 speakers. Even though subject T16 produced the same total WPM as T 19, she produced far higher non-fluency behaviors overall than T19. Not only were WPM and semantic WPM criteria used to judge the fluency so were a high occurrence of hesitation and repairs. Specifically, mean and standard deviation of the occurrences of non-fluency 
behaviors and its sum out of 100 semantic WPM were computed as were WPM and semantic WPM. They are presented in Table V. The results confirmed hypothesis 3 that English-speaking Thai graduate students in this study are unable to simultaneously plan utterances and produce them in a smooth manner. A mean provides the central tendency of the occurrences of each feature as well as WPM and semantic WPM. A standard deviation gives information on the extent to which a set of the number of occurrences varied in relation to the mean. The results show that the 2 fluent subjects' mean scores of non-fluency behaviors are lower than those of the 18 non-fluent subjects. In contrast, those 2 speakers' means of WPM and semantic WPM are higher than those of the 18 nonfluent subjects (see Table V).

\section{TABLE V}

Summaries of Means and Standard Deviations of Fluency and Non-fluency Behaviors

Summaries of Filled Pauses

By levels of Fluency

Variable Value Label

For Entire Population

Mean

Std Dev

Cases

Fluency

.0

8.5914

4.5022

20

Fluency

8.9426

4.4737

18

Total Cases $=20$

5.4309

4.7646

2

Summaries of Unfilled Pauses

By levels of Fluency

Variable Value Label

For Entire Population

Mean

Std Dev

Cases

Fluency

4.3686

3.0772

20

Fluency

.0

4.8048

2.9265

18

Total Cases $=20$

.4426

.2488

2

Summaries of Retraced Restarts

By levels of Fluency

Variable Value Label

Mean Std Dev Cases 


\begin{tabular}{|c|c|c|c|c|}
\hline \multicolumn{2}{|c|}{ For Entire Population } & 7.2657 & 3.8011 & 20 \\
\hline Fluency & .0 & 7.7483 & 3.6890 & 18 \\
\hline Fluency & 1.0 & 2.9223 & 1.1158 & 2 \\
\hline Total Ca & 20 & & & \\
\hline Summari & Unretraced R & & & \\
\hline By levels & Fluency & & & \\
\hline Variable & Value Label & Mean & Std Dev & Cases \\
\hline For Entir & pulation & 1.3326 & .9820 & 20 \\
\hline Fluency & .0 & 1.4315 & .9852 & 18 \\
\hline Fluency & 1.0 & .4426 & .2488 & 2 \\
\hline
\end{tabular}

Summaries of Sum of the occurrences of four features

By levels of Fluency

$\begin{array}{lcllc}\text { Variable } & \text { Value Label } & \text { Mean } & \text { Std Dev } & \text { Cases } \\ \text { For Entire Population } & 21.5583 & 7.4402 & 20 \\ \text { Fluency } & .0 & 22.9272 & 6.4377 & 18 \\ \text { Fluency } & 1.0 & 9.2385 & 3.1511 & 2\end{array}$

Total Cases $=20$

Summaries of WPM

By levels of Fluency

$\begin{array}{lclll}\text { Variable } & \text { Value Label } & \text { Mean } & \text { Std Dev } & \text { Cases } \\ \text { For Entire Population } & 119.86 & 23.11 & 20 \\ \text { Fluency } & .0 & 114.95 & 18.3632 & 18 \\ \text { Fluency } & 1.0 & 164.0909 & 8.3567 & 2\end{array}$

Total Cases $=20$

Summaries of Semantic WPM

By levels of Fluency

Variable Value Label Mean Std Dev Cases

$\begin{array}{llll}\text { For Entire Population } & 97.37 & 22.12 & 20\end{array}$

$\begin{array}{lllll}\text { Fluency } & .0 & 91.99 & 15.5049 & 18\end{array}$

$\begin{array}{lllll}\text { Fluency } & 1.0 & 145.7926 & 1.6648 & 2\end{array}$

Total Cases $=20$

It is obvious that the 2 fluent speakers were capable of exhibiting utterances smoothly without many hesitation and restarts or searching for words or combining them 
into utterances, because the mean of their occurrences of non-fluency behaviors were quite low. T4 and T19 hardly produced unfilled pauses and unretraced restarts. The low occurrences of both features may imply that the 2 subjects were able to execute speech planning and speech production easily and effectively. Also, the evaluator scored their speech production's intelligibility as 3 for T4 and 2 for T19 (see definitions in chapter III). In other words, they had either few vocabulary problems or they were able to handle those problems by filling gaps or eliminating restarts efficiently.

The 18 non-fluent subjects, in contrast, produced those four non-fluency features relatively high out of 100 semantic words. They produced approximately 23 occurrences of filled/unfilled pauses and retraced/unretraced restarts on average. Those high frequencies did interrupt the smoothness and continuity of their utterances, which resulted in decreasing the rater's comprehension of the subjects' messages. These occurrences also reflected that the subjects' L2 production was not automatic or at least partly automatic. The results show that the 18 subjects did not have facility in speech and language performance. The reasons for those occurrences were provided by the subjects in the section of question 2, results and discussion.

\section{Hesitation Phenomena}

The 2 fluent subjects had higher means on filled pauses if compared to the other non-fluency features. From the data transcribed, the use of filled pauses of the 2 fluent subjects tended to be lexical fillers. This supports Riggenbach's research that fluent NNSs may use more lexical fillers than do non-fluent NNSs, who tend to depend more on 
non-lexical fillers and on unfilled pauses. Though subject T4 (fluent) produced a number of lexical filled pauses, she handled her filled pauses interactionally. Those filled pauses, in contrast, acted as a tool drawing more attention of the listener to her speech. In Riggenbach's study the appropriate occurrences of lexical filled pauses performed an interactional function during conversation between two people or more.

Lexical filled pauses (i.e. "like a", "uh", "ah") followed by unfilled pauses (1 second or greater) were more likely to occur when the subjects were searching for the exact words. The non-fluent subjects produced a high occurrence of non-functional nonlexical filled pauses while the 2 fluent subjects rarely did. Additionally, non-lexical filled pauses occurred frequently when the non-fluent subjects were thinking of what to say in order to finish their thought group as in the following:

...I'm getting better in uh.uh. junior and senior and undergraduate cause uh.uh. ...when I was sophomore...

The first " $u h . u h$ " indicates that the speaker was searching for the correct words while the second "uh. uh" was atypical pause in the utterances. It may have meant the speaker was thinking of what to say in English.

\section{Repair Mechanism}

Many of the restarts occurred from the subjects' monitoring of their speech, resulting in grammatically or semantically improved utterances. Generally, if the occurrence of restarts does not interrupt the smoothness of speech, it is argued by some researchers (i.e. Levelt, 1983, Lennon, 1990) that they reflect an ability of a speaker to correct errors. 
...Thai tradition New Year which is, it's the hot, it's considered the hottest in the year...

...people in the north, in the north, northern part of Thailand...

...one term and I find the re-I can solve this problem...

Moreover, a number of occurrences of repetitions occurred frequently in situations where the subjects were attempting to express ideas, thinking to find the exact words or trying to finish their thought group as in the following:

...only Thailand can do than I'm very surprised and and and many country surprise too why...

...the construct like the the the railway...

...the teacher try to uh. (.) try to distribute the the knowledge...

...they they they they don't help you but here they they help me very a lot yeah... ...is very nice, nice, very nice and helpful...

...pay more attention to to the teacher to get more what what the teacher taught us that that is the...

\section{The Combination Between Hesitation and Repairs}

As shown in the data, the 2 fluent subjects barely produced unfilled pauses and unretraced restarts while the others produced chunks of disfluencies. These results may lend support to Riggenbach's (1991) claim that a chunk of disfluencies provides the speakers time to think of what to say next, to repair, or in the case of mixture of filled and unfilled pauses to "analyze/process/observe what has been said and/or what will come next" (p.432). A high number of disfluency chunks indicates nonfluency of the speakers.

The occurrences of hesitation phenomena and repair mechanisms as well as the rate of speech were all markers in this study differentiating high and low fluent speakers. The results indicated that faster speakers exhibited less non-fluency behaviors than slower ones in their utterances. The results computed from the t-test and Man-Whitney 
U/Wilcoxon Rank Sum W Test to increase the validity of those raw frequencies supported the statement above. Two tailed $t$-tests were computed to determine if a difference existed between fast versus slow speech production of English speaking Thai graduate students. Table VI, summarizes this data.

\section{TABLE VI}

Fast Group Versus Slow Group: Hesitation, Repair, The sum of Hesitation and Repair, The speech rate

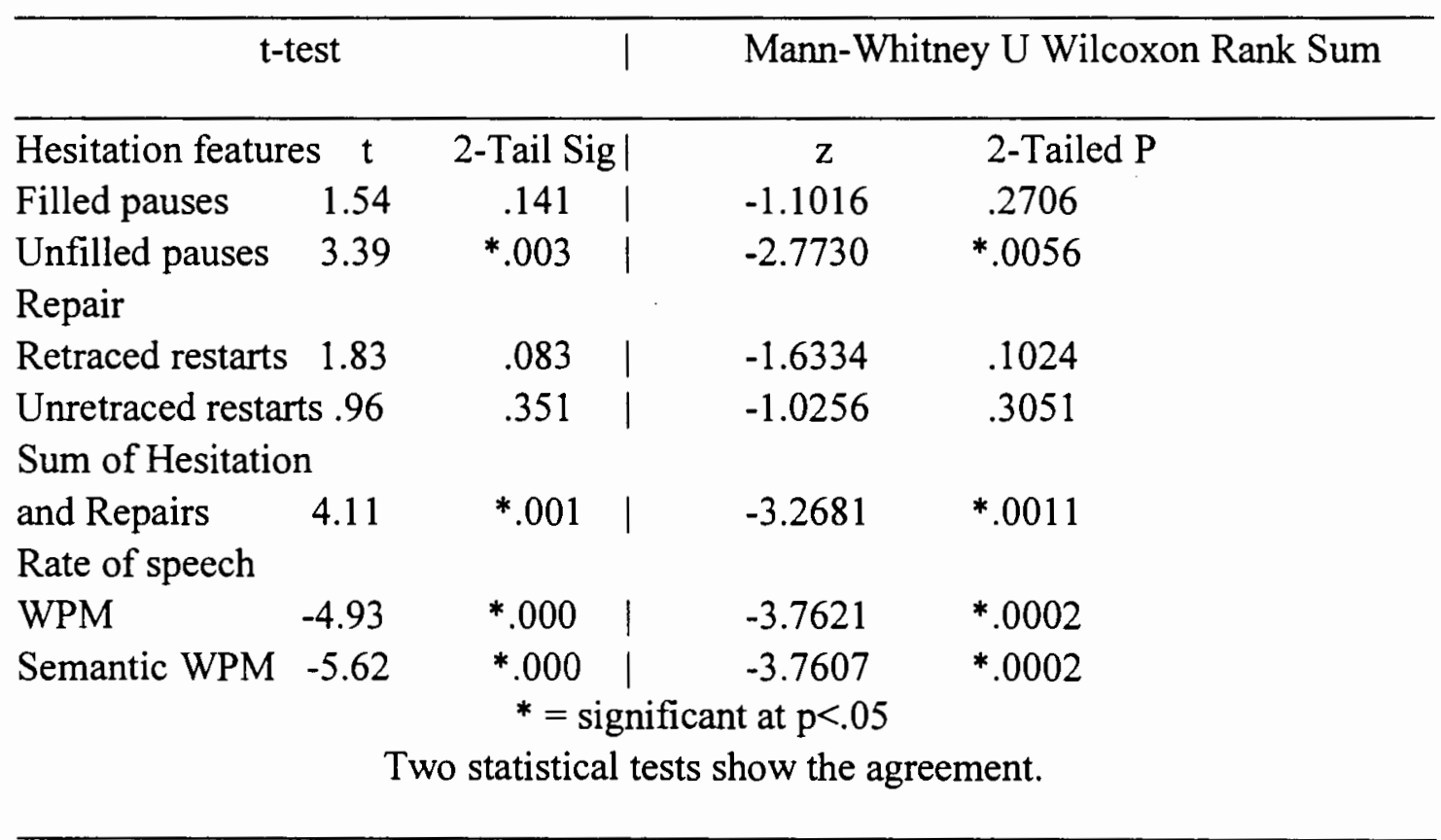

\section{Hesitation Phenomena}

The subjects use of hesitation features suggests that unfilled pauses may be a salient feature in determining the fluency level of speakers in terms of the rate of speech. Additionally, there was a significant difference between group means for the frequency of unfilled pauses from two statistical tests (t-test $\mathrm{p}=* .003<.05$ and Mann- Whitney $\mathrm{U}$ Wilcoxon Rank Sum $\mathrm{p}=* .0056<.05$ ). Differences were revealed in the kind of 
unfilled pauses used by the fast group versus the slow group. Although both groups produced unfilled pauses in midclause, the use of unfilled pauses of the fast group tended to occur when they were searching for vocabulary while the slow group produced those nonfunctional pauses not only for that reason but also paused while formulating their thoughts and language all of the time and often paused without reasons. For the filled pauses, the differences were not statistically significant.

\section{Repair Mechanism}

Differences between the subgroup were not statistically significant for repair mechanisms.

\section{Sum of Hesitation and Repair}

The fast group $(n=9)$ produced fewer non-fluent behaviors than the slow group $(n=11)$. The study offered a logical explanation that the fast speakers who were capable to plan and utter L2 simultaneously spent little time in their speech execution. One explanation for this behavior may be that these two subjects often interact verbally with NSs. Practice speaking English derives from the opportunities for contact between subjects and TL speakers. It was deduced from the demographic data, that having social interaction with NSs frequently benefits L2 speakers, as NSs provide a valuable source of L2 input. This is done in such a way that the subjects may not only expand their vocabulary repertoire but also improve vocabulary comprehension. Their utterances were quite smooth and the occurrences of hesitation and repair features were relatively low. 
The results confirmed that there was strong evidence that the high frequency of the sum of hesitation and repairs were related to the subjects' rate of speech.

\section{$\underline{\text { Rate of Speech }}$}

There were significant differences again in WPM and semantic WPM. The comparative rates of speech suggest a clear pattern from fast to slow. The 9 subjects of the fast group produced significantly higher WPM and semantic WPM. Their hesitation and repair features out of 100 semantic words were less than the 11 subjects of the slow group. It was apparent that the speakers who produced WPM and semantic WPM faster were able to speak more fluently as measured by the $t$-tests (mean difference $=-34.3202$, $p=* .000)$. The fast speakers had less problems in producing L2 at a significant level (mean difference $=10.1482, p={ }^{*} 001$. Considering the number of occurrences of nonfluency features, however, only 2 subjects in the fast group appeared to be fluent. Interpreting these WPM and semantic WPM counts require a consideration of the quality of the talk also.

\section{SUMMARY}

These results are consistent with Riggenbach's research (1991) showing that the rate of production of unfilled pauses by non-fluent speakers was significantly higher than the rate produced by the fluent speakers. Also non-fluent speakers spoke significantly slower than the fluent speakers during the interview. The data collected during the interview supported three hypotheses: 
1. Listeners understand approximately $80 \%$ or less of the subjects' messages due to errors in pronunciation, grammar, fluency, or vocabulary that occasionally interfere with intelligibility.

2. Speakers' encoding capacity particularly in lexis significantly influences the fluency level of their spoken English.

3. English-speaking Thai graduate students in this study are unable to simultaneously plan utterances and produce them in a smooth manner.

The score rates of 20 subjects from the interview suggested that the listener may not have comprehended the subjects well because of either their pronunciation, ungrammaticality or non-fluency. T19 was rated as fluent; however, less than $80 \%$ of her messages were understood due to her strong Thai accent. The results show that fluency is not the sole factor that is related to speakers' intelligibility. Fluency is more related to speakers themselves which was the focus of this study. T2 and T14 appeared to be rated high in terms of their intelligibility because they both spoke English with an English accent. But their speech production was not in line with the notion of fluency because they both had lexical searching problems and had syntactic difficulty while formulating their thoughts and language all the time. The intelligibility of their messages may come from the evaluator's guess from some words she heard. In contrast, T19 was able to connect sentences in stretches of discourse and form a meaningful whole out of a series of utterances (Brown, 1987). If the rater had been more accustomed to a Thai accent, she might have been better able to understand the whole message of T19. 
The results confirmed that all of the Thai graduate students in this study have problems with lexis. Though $\mathrm{T} 4$ and $\mathrm{T} 19$ reported their searching vocabulary problems as the other subjects did, T4 often demonstrated communication strategy, circumlocution, or giving an example to help the interlocutors understand her messages rather than being silent or filling the gaps by non-lexical filled pauses or restarting her sentences all the time. T19 had few problems in planning thoughts and uttering them in L2 simultaneously; she indicated that she has spoken English routinely for the past 3 years that she has been in the United States. Larsen-Freeman and Long (1991) state that the number of years of exposure contributes greatly to communicative fluency. NNSs are supposed to have chances to practice by using the language with NSs in a real communication (Prokop, 1989). T4 and T19 had spent more time in the US than the other subjects. They reported in their demographic data that they both were High Input Generator (HIG) type of L2 learners. HIG learners are described by Seliger (1977) as eager to put themselves into the position of interaction initiator in order to use the opportunity to talk. While subject T4 was a student in the United States, she was the only Thai student in class. She still keeps in touch with her American friends and host family frequently. T4 emphasized that informal verbal practice with NSs has improved her English fluency. T19 revealed that she intentionally avoided being with her community and speaking $\mathrm{L} 1$ all the time because she realized that having conversation with NSs allowed her to notice a gap between what she produced and what is produced by speakers of the L2, which helped expand her vocabulary. 
On the other hand, the other 18 subjects reported that they seldom spoke English within a day neither in nor outside the classroom. They did not have American friends that they could talk to on a conversational level. They clung to their Thai friends and colleagues almost all of the time. Some said that they wanted to have at least one American friend. The others Thai students preferred staying with American host families. These factors may have affected their non-fluent speaking behaviors. 


\section{CHAPTER V}

CONCLUSIONS

\section{SUMMARY OF THE STUDY}

This study examined the English fluency of Thai graduate students in the United States. The notion of fluency is strictly limited to three features discussed thus far: hesitation, repair, and rate of speech. Fluency is the most important aspect of how well the subjects can express their thoughts and produce those thoughts in the L2 because only fluent speakers in this study were able to convey the entire intended messages smoothly and effortlessly. The results verify that Thai graduate students who were the subjects here believe they have communication difficulties because of their limited command of vocabulary and the incapacity to express sufficiently in English what they are thinking in Thai. In fact, it may be more complicated as any students who have difficulty in a second language may blame their problems on vocabulary.

With the evidence from both qualitative and quantitative analyses, including the subjects' demographic data, it was demonstrated that Thai graduate students in this study have little social interaction with speakers of the TL, which decreases their opportunities to practice speaking English. This lack of interaction hinders the development of their fluency because they have an insufficient source of L2 input from speakers of the TL, thus reducing their $\mathrm{L} 2$ development. 


\section{IMPLICATION FOR THAI GRADUATE STUDENTS \\ IN THE UNITED STATES}

The study yields negative results, in that, Thai graduate students were unable to simultaneously plan utterances and produce them in a smooth manner. This section of the paper further considers some ways of helping other Thai graduate students who have non-fluency in English and want to overcome those problems.

The first hypothesis showed that the trained instructor still had difficulty in understanding the subjects' messages. It is likely that someone who has no training in working with NNSs would have even more difficulty. For example, he/she might not be able to identify the detailed messages the main ideas but only some words. Listener's comprehension could be increased if the speakers paid more attention to clarifying ideas and pronunciation. The subjects' retrospection in the second research question showed that the subjects were not overly concerned with their pronunciation and clarity of meaning. Working on these two dimensions could help increase listeners' comprehension of L2 speakers' messages.

In addition, the results above suggest that the subjects have language communicative needs. The third hypothesis supports this idea because of the subjects' lack of capacity to simultaneously plan utterances and produce them in a smooth manner. Because most of the subjects have fairly high TOEFL scores, they were not required to study the English language. Additionally, because of their majors, they mainly focus on their academic success. They spend little time practicing speaking English. It is possible 
that there are no sufficient out of class activities for speaking English. The subjects may not interact with NSs for their communicative needs. The frequency of practice does affect the subjects' fluency level. It implies that practicing English more often helps facilitate subjects' effort in speaking. The more frequently L2 learners practice, the more reflexive they become in producing $\mathrm{L} 2$.

Moreover, the interview results showed that while the subjects were trying to express their intended messages, the interviewer frequently guessed what they were thinking and was the person who conveyed the subjects' ideas instead. This supports Pica's idea (1987) that low fluent L2 speakers are given limited opportunities to modify their output as NS interlocutors often tend to correct them. NNSs then need only to acknowledge the model provided rather than attempt to produce their own output towards targetlike use. Therefore, the participatory role of respondent seems to belong to NNSs in the conversation. Also, they are more passive and more willing to yield to NSs the role of discourse initiator. Due to NNSs' roles as foreigners and respondents in the communication, "this will influence reactions to what can be described as parameters of fluency" (Sajavaara, 1987, p.58).

Similarly to Pica, Sajavaara (1987) points out that foreigner talk is likely to take place in communicative situations between NSs and NNSs. It is the nature of the adjustments NSs make when conversing with NNSs and these modifications that affect SLA. "The conversational mechanisms which govern the interchange of textual roles, and the turn-taking system, may not function in the same way they function in full-scale 
L1 situations" (Sajavaara, 1987, p.58). For instance, NNSs are provided more time to formulate and finish their messages. Also, NSs tend to give reformulation to NNSs' sentences during and after hesitations and pauses.

\section{STRATEGIES}

\section{IN DEVELOPING FLUENCY}

Strategies for development of fluency may aid not only instructors who want to help their ESL students to get rid of foreigner talk but also foreign students who want to overcome their linguistic barriers. Fluency will be developed by using the following strategies. First, according to the results of this study, lack of vocabulary is one of the biggest obstacles for Thai graduate students in achieving fluency in English. Developing vocabulary strategies (Smith et al. 1992) can help students because they are not going to learn all of the words in English. Rather, they should attempt to communicate their idea by learning the strategies themselves and suggestions for doing this are included in the brochure (see Appendix E, p. 107). Though they may not know the exact word, they can use vocabulary strategies to help them sound fluent. This strategy is one of the communication strategies called paraphrase. When L2 speakers cannot think of the exact words, paraphrase them. There are seven ways (Smith et al, 1992, p.149-150) they can choose.

1. Analysis (a toaster)
Break the object down into its parts. "I can't think of the word, but it has two openings where you put in bread to cook, a long electrical cord, something on one end that you push down to make it work, and a piece of metal to adjust the temperature."

2. Cause and effect Describe what causes a phenomenon or what results 
(gravity) from it.

"I'm not sure what the word is, but this is what makes objects fall toward the earth."

3. Example Give an example, we tend to believe that all Spaniards (stereotype) play the guitar and dance flamenco."

4. Comparison and Talk about how the object is similar to or different Contrast from something else.

(a sweet potato) 'I don't know what it's called in English, but this vegetable is similar to a potato in shape and how it is grown, but is different from a potato because it is usually deep orange and has a sweet taste."

5. Definition (a shopkeeper) Use a definition to describe what you mean. "The word I'm looking for means a person who owns and works in a small store."

6. Opposites If you can't think of the word, use its opposite. (sad) "I'm not exactly sure of the word, but it means that a person is unhappy."

7. Description (a razor)

Give the size, color, shape, material, function, or other aspects of the object.

"I'm talking about a small object with a handle with a handle that is made of metal or plastic. One part of it is flat and silver colored and is very sharp. It's used to take the hair off a person' face or body. Can you tell me the word in English?"

Second, as shown in Chapter IV, hesitation phenomena (filled and unfilled pauses) is another problem for the subjects in this study. Hesitation phenomena frequently interrupt the smoothness of utterances, resulting in problems of listeners' understanding. There are two means L2 learners might want to consider using to eliminate those pauses, gambit and lengthening.

A gambit is the use of interpersonal remarks in order to preserve the smoothness between conversational partners (Edmondson \& House 1981) as well as to provide the speaker extra time for the production process. (e.g., What I would like to say is ...). Gambits can be employed in order to eliminate filled pauses. Another strategy, 
lengthening, is an effort to maintain transition smoothness by drawing a phonetic segment of the first word in order to avoid an unfilled pause (e.g., c-o-m-e back tomorrow, a-f-t-e-r you finish your report). These strategies can assist students in avoiding using filled and unfilled pauses because they provide speakers extra time to think of what to say next, the correct word or how to finish their thought.

Third, this study reported that non-fluent subjects produced a higher volume of retraced and unretraced restarts than the fluent subjects. Quite often, restarts occur when the subjects began sentences that they did not finish or if they made several attempts to say a word or to express an idea. There are three ways to handle these problems: island of reliability (Lesser et al. 1977), successful false start and using a phrase such as "Let me start over", "Let me start again" and "Let me begin again."

An island of reliability is a stock phrase of a speaker. When a speaker has problems, he/she decides to use their stock phrases as a "safe island" such as "and" or "but" (e.g., They asked me to get some music- and - well I don't know.) Successful false start is the breaking off and reformulation of a sentence exhibiting no marked reduction of smoothness (e.g., I think you should/ the best thing would be to buy a new car). A. speaker can use a phrase such as "Let me start over", "Let me start again" and "Let me begin again" when having problems in organizing the sentence. The speaker finishes the repair sentence first, and then paraphrases what he/she wants to say, then, starts the sentence again with one of the three phrases mentioned above. 
TABLE VII

Breakdown in the Production Process

at Utterance Level (Rhode, 1985)

\begin{tabular}{|l|l|}
\hline Strategies to eliminate nonfluency manners & Nonfluency manners \\
\hline Paraphrase or Vocabulary strategies & Lack of vocabulary \\
\hline Gambit & Filled pauses \\
\hline Lengthening & Unfilled pauses \\
\hline Island of reliability or using some phrases & Repetition \\
\hline Successful false start & Problematic false start \\
\hline
\end{tabular}

Additionally, these results support my original hypotheses and reinforce the need for pre-departure and in-country orientation to Thai students to make maximum use of their sojourn aboard. The orientation should offer advice on behavior modification to benefit L2 language capabilities during their stay in the TL environment. Perhaps these guidelines could be presented in the form of written material to all students who apply for a student VISA at the U.S. embassy. As a result of the study, the researcher has designed a sample brochure that may help improve Thai graduate students motivation in developing fluency in a practical sense. It is presented in Appendix E. Additionally, a workshop for ESL instructors in teaching fluency is provided in Appendix F.

\section{LIMITATION AND}

\section{METHODOLOGICAL PROBLEMS}

There are some limitations in this study. Due to the small sample size, the results should be reserved to this particular group of subjects. In addition, this study aimed at investigating only three features: hesitation, repair, and rate of speech in evaluating the subjects' speech production in L2. The results show that there were only two subjects out of twenty rated as fluent because of their capacity of producing smooth utterances along 
with a relatively high rate of speech and a relatively low pause count. These features are factors that appear to be tied strictly to the notion of fluency without consideration of other factors such as those which enable NNSs to overcome their communication difficulties like interaction between a speaker and interlocutor. In other studies, different conditions have to be met in order to determine the fluency level of NNSs such as grammar and pronunciation. If this study took those factors into account, the results might be different. However, with this limitation in mind, this study addresses a link between the process of speech planning and speech production in L2 of NNSs. The results show lack of communicative strategies of the subjects to give themselves extra time to think what they would say in their L2 to match with what they would say in their native language.

\section{SUGGESTIONS FOR FURTHER RESEARCH}

The study provides a greater understanding of fluency phenomena and its measurable features. As a result, the researcher identified some strategies for assisting L2 speakers in counter-balancing non-fluency problems. The investigation of the relative effectiveness of communicative strategies that actually counterbalance nonfluency behavior or are considered compensatory fluency should be examined for further research. Also, another research question on English written fluency should be investigated. What features in writing of NNS may contribute to the judgments of written fluency? Are there any strategies that can help NNSs counterbalance non-fluency writing problems? 


\section{REFERENCES}

Arevart, S., \& Nation, P. (1991). Fluency improvement in a second language Regional English Language Center, 22 (1), 84-93.

Bamford, J. (1989). Comments on Elizabeth Gatbonton and Norman Segalowitz's "Creative automatization: Principles for promoting fluency within a communicative framework". TESOL Quarterly, 23 (2), 363-373.

Beebe, M. L. (1988). Issues in second language acquisition: Multiple perspective. NY: Newbury House Publishers, a division of Harper \& Row, Publishers, Inc.

Brown, H. D. (1980). Principles of languages learning and teaching. Englewood Cliffs, N.J.: Prentice-Hall, Inc.

Carroll, B. J. (1980). Testing communicative competence: An interim study. Oxford: Pergamon.

Clahsen, H. (1987). Natural language development: Acquisitional processes leading to fluency in speech production. In H. W. Dechert \& M. Raupach (Eds.), Psycholinguistic models of production (pp.45-65). Norwood, NJ: Ablex.

Edmondson, W., \& House, J. (1981). Let's talk and talk about it. Munchen: Urban and Schwarzenberg.

Ely, M. C. (1986). An analysis of discomfort, risktaking, sociability, and motivation in the L2 classroom. Language Learning, 36 (1), 1-26.

Faerch, C., \& Kasper, G. (1983). Plans and strategies in foreign language communication. In C. Faerch \& G. Kasper (Eds.), Strategies in interlanguage communication (pp. 20-60). London: Longman.

Fathman, K. A. (1980). Repetition and correction as an indication of speech planning and execution processes among second language learners. In H. W. Dechert \& M. Raupach (Eds.), Towards a cross-linguistic assessment of speech production (pp. 77-87). Frankfurt am Main, 
Federal Republic of Germany: Peter Lang.

Fillmore, C. J. (1979). On fluency. In C. J. Fillmore, D. Kempler, \&

W.S.-Y. Wang (Eds.), Individual differences in language ability and language

behavior (pp. 85-105). NY: Academic.

Gardner, R. C., \& Lambert, W. E. (1972). Attitudes and motivation in second language learning. Rowley, Massachusetts: Newbury House Publishers, Inc.

Gass, M. S., \& Varonis, M.. (1994). Input, interaction, and second Language production. Second Language Acquisition, 16 (3), 283-302.

Gelderen, A. V. (1994). Prediction of global ratings of fluency and delivery in narrative discourse by linguistic and phonetic measures- oral performances of students aged 11-12 years. Language Testing, 11 (3), 291-317.

Hieke, A. E. (1985). A componential approach to oral fluency evaluation. The Modern Language Journal, 69 (2), 135-142.

Ioup, G., Boustagui, E., Tigi, E. M., \& Moselle, M. (1994). Reexamining the critical period hypothesis: A case study of successful adult SLA in a naturalistic environment. Second Language Acquisition, 16, 73-98.

Krashen, S. (1981). Principles and practice in second language acquisition. Elmsford, NY: Pergamon Press.

Kurian, G. T. (1992). Encyclopedia of the third world. (4th ed.). New York: Facts on file.

Larsen-Freeman, D., \& Long, M. (1991). An intreduction to second language acquisition research. London: Longman.

Lennon, P. (1990). Investigating fluency in EFL: A quantitative approach. Language Learning, 40 (3), 387-417.

Lesser, R. V., \& Erman, D. L. (1977). A retrospective view of the Hearsay-II architecture. In proceedings of the 5th International Joint Conference on 
Artificial Intelligence, Vol. II. Cambridge, Massachusette: MIT.

PP. 790-800.

Levelt, W. J. M. (1977). Skill theory and language teaching. Studies in Second Language Acquisition, 1, 53-70.

Levelt, W. J. M. (1983). Monitoring and self-repair in speech. Cognition. 14, 41-104.

Mclaughlin, B., Rossman, T., \& Mcleod, B. (1983). Second language learning:

An information-processing perspective. Language Learning, 33, 135-157.

Pica, T. (1987). Second-language acquisition, social interaction, and the classroom.

Applied Linguistics. 8, 3-21.

Rehbein, J. (1987). On fluency in second language speech. In H. W.

Dechert \& M. Raupach (Eds.), Psycholinguistic models of production (pp. 97-105).

Riggenbach, H. (1991). Toward an understanding of fluency: A micro analysis of nonnative speaker conversations. Discourse Processes, 14 (4), 423-443.

Rhode, L. (1985). Compensatory fluency: A study of spoken English produced by four Danish learners. In E. Glahn \& A. Holmen (Eds.), Learner Discourse (pp.43-70). Copenhagen, Denmark: B. Stouggard Jensen.

Sajavaara, K. (1987). Second language speech production: Factors affecting fluency. In H.W. Dechert \& M. Raupach (Eds.), Psycholinguistic models of production (pp. 45-65). Norwood, NJ: Ablex.

Sajavaara, K., \& Lehtonen, J. (1980). The analysis of cross-language communication: Prolegomena to the theory and methodology. In .H. W. Dechert \& M. Raupach (Eds.), Towards a cross-linguistic assessment of speech production (pp. 55-77). Frankfurt am Main, Federal Republic of Germany: Peter Lang.

Schmidt, R. (1992). Psychological mechanisms underlying second language fluency. Studies in Second Language Acquisition, 14, 357-385. 
Schneider, W., Dumais, S., \& Shiffrin, R. (1984). Automatic and control processing and attention. In R. Parasuraman \& D. Davies (Eds.), Varieties of attention (pp. 1-27). Orlando, FL: Academic.

Seliger, W. H. (1977). Does practice make perfect?: A study of interaction patterns and L2 competence. Language Learning, 27 (2), 263-278.

Starkweather, W. C. (1987). Fluency and stuttering. Englewood Cliffs, NY: Prentice Hall, Inc.

Smith, J., Meyers, M.C., \& Burkhalter, J.A. (1992). Communicate: Strategies for international teaching assistants. Englewood Cliffs, NJ: Regents/ Prentice Hall.

Speaking Proficiency English Assessment Kit (SPEAK) Test. (1982).

Educational Testing Service, Princeton, NJ. (Produced by the Test of English as Foreign Language Program.)

Spolsky, B. (1977). The comparative study of first and second language acquisition. In F. R. Eckman \& A. J. Hastings (Eds.), Studies in first and second language acquisition (pp. 167-184). Rowley, Massachusetts: Newbury House Publishers, Inc. 
APPENDIX A

DEFINITION OF EVALUATION SCORES 


\section{APPENDIX A \\ DEFINITION OF EVALUATION SCORES}

ITA TEST SCORE SHEET

Pronunciation $\quad 0|.5| 1|1.5| 2|2.5| 3 \mid$

Grammar $\quad 0|.5| 1|1.5| 2|2.5| 3 \mid$

Fluency $\quad 0|.5| 1|1.5| 2|2.5| 3 \mid$

Comprehensibility $\quad 0|.5| 1|1.5| 2|2.5| 3 \mid$

Each score is defined as:

Pronunciation

3- Nativelike stress and intonation patterns. Accurate pronunciation and clear enunciation of individual sounds. Almost always intelligible.

2- Minor problems with stress and intonation. General accuracy in pronunciation of most individual sounds. some difficulty with enunciation, but is generally intelligible.

1- Major problems with stress and intonation. Difficulty with the pronunciation of some individual sounds. Poor enunciation. Occasionally unintelligible.

0 - Nonnative stress and intonation patterns, poor pronunciation of individual sounds, or lack of enunciation cause general unintelligibility.

\section{Grammar}

3- Accurate grammatical form and appropriate use of structures. Occasional errors that might be made by a native speaker.

2- Occasional difficulty with accurate grammatical form and appropriate use of structures. Minor errors that do not interfere with meaning.

1- Frequent difficulty with accurate grammatical form and appropriate use of structures. Major errors that sometimes interfere with meaning.

0 - Constant difficulty with accurate grammatical form and appropriate use of structures. Only memorized phrases can be easily understood.

\section{Fluency}

3- Very few nonnative pauses. Smooth and nativelike rhythmic patterns that never interfere with intelligibility.

2- Some nonnative pauses. Some difficulty with smooth and nativelike rhythmic patterns, which causes occasional interference with intelligibility.

1- Numerous nonnative pauses. Much difficulty with smooth and native-like rhythmic patterns, which causes frequent interference with intelligibility.

0 - Nonnative pauses and halting rhythm causes constant interference with intelligibility. 
Comprehensibility

3- Completely comprehensible. Only a few errors in pronunciation, grammar, fluency or vocabulary.

2 - Generally comprehensible. Some errors in pronunciation, grammar, fluency or vocabulary that occasionally interfere with intelligibility.

l - Somewhat comprehensible. Major errors in pronunciation, grammar, fluency and vocabulary that often interfere with intelligibility.

0 - Generally incomprehensible due to very little ability in pronunciation, grammar, fluency or vocabulary.

* Comprehensibility scores were used primarily as the overall scores of listeners' understanding. 
SGSFYHd YO SGYOM (JILNVWGS)

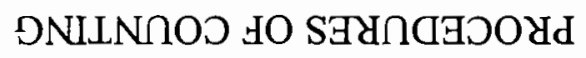

G XIGNGddV 


\section{APPENDIX B \\ PROCEDURES OF COUNTING \\ ( SEMANTIC) WORDS OR PHRASES}

I. Sounds are not recognized as words.

" $s "$ sound

II. Reformulations in which part of the original utterance are repeated are not recognized as words.
$a$-academic
be-because
Bri-British
fam-family
grand-grandparent
in reli-in religion
$k$-come
peop-people
pers-person
posi-position
pre-presentation
semes-semester

III. Reformulations in which part of original utterance are rejected are not recognized as words

...who con like the government

...only a lit a few people

...to my grandfa grandmother's house

IV. Each abbreviation is recognized as one word.

the United States

English Language Institute $(E L I)$

Los Angeles $(L A)$

Grade Point Average (GPA)

V. Each following word is recognized as one word.

cannot

there's

it's

uh-huh

they're 
I'm

VI. Subjects' slip of Thai proclamations, words or phrases are not recognized as words.

VII. Lexical filled pauses are recognized as words but not as semantic words because they contribute little or no semantic information in context. However, there are some of them that provide meaning, for example, "like" contributes "as" or "for example" meaning.

...some sense of me it's kind of like um. (.) what's a word, kind of (.)...

...because like in Thailand, like we have to respect the instructor and things like that...

...but I saw the most people have a job have a (.) like a (.) good standard of living...

...going to some group of people not spread of well the rich people is the truth...

...and if people is you know like well-educated they won't like this guy...

...I see in Thailand the economic like having a highly dependent on the politics so it's kind of um.

VIII. The occurrences of repeats of one words or greater in twice times are recognized as one restart but if triple, recognized as twice restarts. 
APPENDIX $\mathrm{C}$

SAMPLES OF TRANSCRIPTS 


\section{APPENDIX C}

\section{SAMPLES OF TRANSCRIPTS}

Speech samples of transcripts of four subjects are presented: T3 and T13 considered as nonfluent, T4 and T19 as fluent. Selection of 5 excerpts of each subject were analyzed. Filled pauses were highlighted by bold. Unfilled pauses of a second or greater were indicated by (.). Retraced restarts were italic and unretraced restarts were underlined.

T3

I: What is your name?

T3:

I: What are you studying here in your university?

T3: Uh. Chemical Engineering (.) and (.) this is my major and my minor is a [untranscribable words] most of my minor subject concern about the uh. environmental engineering.

I: Are you enjoying the courses you are taking?

T3: yes yes very much but I have ah. ah. hum. the first intention to study in America and (.) and I find out some (.) uh. some information that uh. This university pretty good especially for the Chemical Engineering department, there are, they have uh. there are about a lot of um. famous professor and also the research subject that I also interest

I: So, it's that why you choose this state, instead of another states or another countries to study?

T3: um. the reason that I choose this State I have the three reasons. First is the $a$ academic rank o.k. This state Even though it's not a top ten rank but uh. it's (.) the rank is twenty nine and ah. the second is the surrounding ah. I heard from (.) ah. a lot of my friend also my father he has been here since ah. nineteen sixty he study in the Texas but er. he has er. some of his friend study here he [an untranscribable word] to me, and my friend [an untranscribable word] to me weather nice people pretty good uh. everything I can find it here not only inside the class but also outside the class I can enjoy and also safe for me to ah. compare to the big city in this city it's pretty good and the third reason is I choose here is the o.k. cost of living and expense is not so expensive compare between here and ah. (.) California. pretty, pretty different

I: When you first came here and experience your classes, how did you have to change your self-study habits? 
T3: First $I I$ like to explain in this way in Thailand we can take a lot of class for each term or each quarter oor (Thai proclamation) in Thailand we have the semes-semester system but here er. we have the system is the quarter and here there's a lot of homework each subject in Thailand o.k. I can take five or six or maybe up to seven class each semester but (.) uh. in in here I just only take the maximum is three classes and ah. the way that I uh. prepare to change from uh. my system to this system is to keep on to study uh. maybe some it very important to read before go inside class and after (.) uh. study immediately maybe in that evening ah. try to review and study make a note or something like this cause sometime (.) I cannot take a note right away cause uh. teacher or professor speak very fast but I just uh. short note so I have to go back home and (.) uh. pull out that short term to be the full detail uh. understanding.

I: You should do just fine. When you first came to United States, what surprised you?

T3: (.) people first but uh. when I look through the window from the (.) aircraft uh. (.) uh. compares between Thailand here er. when I saw the people all of the people around me the American people but ah. big people Thailand uh. we usually have o.k. size like me not too tall um. but all here all around s-American people speaks English all the time so (.) I very exciting

I: Tell me a little bit about some tradition in Thailand, for example, America has a Halloween, Christmas's day, or Valentine's day, how about in Thailand?

T3: In Thailand we we have a kind of various traditional (.) o.k. may may uh. we even though we don't have a Christmas but also we celebrate Christmas in Thailand but the actually the traditional maybe New Year for Thailand it's er. it's round April we we call Song Kran uh. (.) the type of the weather in Thailand, ve-er. it's very hot so (.) when we have the Songkran it mean New Year comes coming we sometime we have the custom that we (.) use (ps) (.) water play with the water maybe uh. (.) what can I say if the traditional in in we have the (.) good traditional when we er. we o.k. keep go to find some some father er. find my mother my ah. father and mother or my cousin I use the water to cheer him up or something like that

I: What are you the most proud of in your country?

T3: Most proud (.) (ps) I think uh. (.) Thai ah. (.) the word Thai means we (ps) (.) independent we (.) uh. we have never been (.) uh. under (.) under rule for example India uh. English England take charge tor [a Thai word] for many year and use they change uh. their official language to be the English one but Thailand even though we (.) we (.) no have ah. some kind like that o.k. uh. some some people say o.k. for the civilized, civilization country when wey when they came came to take over that (.) uh. develop country they bring the technology bring the knowledge but uh. in my opinion even though we do not have that kind of uh. new technology but we very proud that we (.) independent not ah. (.) under er. (.) under some countries some France, Portuguese and English England and we have uh. we have the (.) kings that ah. we call Rama the fifth he was very 
clever when French and England (.) k-come to our country maybe they can claim that uh. we not ah. civilize country we have uh. good (.) study (.) system we not have some civilize thing that they have so they try to take in charge but uh. but uh. (.) that king sent his son, sent sometime, sent his daughter to go oversea to study and bring the technology and knowledge to develop. 
T4

I: What is your name?

T4:

I: $\quad \overline{\text { And what }}$ are you studying here?

T4: En.Environmental Engineering

I: Are you enjoying your study?

T4: so far yes.

I: Can you tell me al little bit about what you're studying, your area?

T4: um. my area environmental engineering basically it's like about um. (.) you know all the power engine environment how you take care of them like how you um. like control the level of in air air pollution or water pollution stuff like that

I: Why do you choose here, not the other states, or another countries, like England or Australia?

T4: oh! well I guess I am kind of um. um. accustomed United States and I have friends and family I mean like my aunts here in United States and my boyfriend going to school in uh. Washington (.) so this is like uh. school near my family, my boyfriend stuff like that and it's a good school.

I: When you first came here, how did your adjust your study habit?

T4: um. let's see my study habit? um. (.) well I don't know just like (.) just like nothing to adjust, I guess.

I: How long have you been here?

T4: in here um. like ten weeks, I was here like in um. November, No, November.

I: Is this the first time you're studying in United States?

T4: um. no, um. I did my degree here in a U.S university.

I: $\quad$ So, the system, you think the change between Thai education system and American education system wasn't difficult?

T4: for me um. no. no. I don't think so (.) just one thing that I need to adjust like uh. the system the quarter system the semester system because in Thailand we have semester system here, I'm having a quarter system, which is like very fast

I: What surprise you the most when you first came?

T4: um. this time?

I: When you first came.

T4: My first came here it's like long long time ago (laugh) it's like um. ten, eleven I was in New York and I think I like oh! my god like downtown, skyscraper and all the stuff and uh. elevator and a you know real fast like wow! and um. what else (.) like um. subway (laugh) yeah, we don't have that in Thailand.

I: Tell me about some tradition in Thailand, for example, United States has Halloween, Christmas, Valentine's day.

T4: o.k. um.(.) we have um. (.) Songkran this is my favorite one because it's like in the middle of April which is very hot there and um. like three day festival. People can throw water at each other and all like get soak all day (laugh).

I: $\quad$ So you have a lot of fun especially. 
T4: yeah. Yeah sometime we put like a big bucket water on the truck and drive around the town and splash water at everybody (laugh).

I: all right, what do you most proud of in your country?

T4: In my country um (.) Well (laugh) what I most proud of um. I think um. (.) the way we treat um. older people like in here you know like um (.) we have like very high family value there we have to take care our mom, our grandmother when they get old, and sending, sending them to nursing home. It's not like not com, common in our country we not do that.

I: What do you dislike most in America?

T4: um. (.) let's see um. what I dislike the most. I don't know (laugh) I cannot think of one right now.

I: Do you think there is any corruption in your country?

T4: yes a lot.

I: What can you tell me about it? What do you think about it?

T4: well, I don't like it but then I think it's very difficult because the people who are corrupting they're in high position, they can you know easily cover up what they did like um. just one guy there was an election in Thailand like last year and this guy who's like buying vote and the police even caught his um. what is it like his supporters and with a lot of money and you know and everybody knew that he did it but then he was elected into the cabinet because he he bought votes and then the thing just you know gone (laugh) nobody talk about it anymore.

I: What can you do to help solve this problem?

T4: what can I do? It's very difficult. I think um. the first thing we need to do in Thailand is to give people more education so then they can sort of what's right, what's wrong and if people is you know like well educated they won't like this guy and if he is not in the posi, if he doesn't have position the cabinet right now um. the police will you know have the easier access to to this thing.

I: $\quad$ There are a lot of Thai students now coming to study in America and perhaps you broke your tradition of living with your family when you came here and you probably have a lot more freedom, do you think that this freedom that you experience here will change your attitude towards your culture when you go back to Thailand?

T4: um. I don't think so because um. yeah. I admit that I do have more freedom here but um. when I go back to Thailand I just you know become the same person I was before like not not exactly the same but you know I obey rules in the house, like now I have no curfew, no nothing but then when I go back home, my mom still you know um. tell me what time to to be home stuff like that and I still obey her

I: Do you think do you have inner resent with about having to do that?

T4: um. no, because before I came here I, I knew my option that when I'm here, I can, you know, um. what is it like, um. (.) I can live like American, yeah, almost like that and . have a control of myself when I go home it's like the Thai tradition that we have to listen to mom stuff like that, yeah 
I: I like to talk to you after you get home about a year, see what it's like?

I: What is economic situation like in Thailand, it's like compared to United States?

T4: Economic, um. (.) I see in Thailand the economic like having a highly dependent on the politics so it's kind of um. (.) when the political situation is good the economic Thai is good also but right now I'm I am not I don't know I haven't been updated with the news from Thailand yet but I think um.(.) see in United States having problem also the federal shut down whatever it is um. it's very difficult for me to compare right now I think they have both have problem but um. different types of problems.

I: $\quad$ Have you heard about the Yandra monk who violated the monk regulation by having intercourse with women?

T4: yeah.

I: What do you think about him?

T4: well, I think um. (.) like I said before that in Thailand people who in the higher position they can cover (ps) what what they did wrong and this guy like he is kind of powerful right now he's not in Thailand anymore. He is in LA. I think and you know because actually um. Thai government like ban his visa and everything but however he still can you know escape can left the country.

I: Do you have any you carry with you in your mind proverb or value that you believe in that you think of sort of help you with your life here

T4: here?

I: or there?

T4: not really just live like day by day

I: Is that a value?

T4: I guess so.

I: Do you think do you brought much culture here?

T4: here um. (.) yes, I think yeah

I: like?

T4: like um. the way of living stuff like that, still call my mom (laugh) like every other day and what else um. (.) I don't know, food still eat Thai food. 
T13

I: What is your name, what are you studying in here?

T13: I'm, my name is um. uh. I am uh. I am IE student here to graduate

I: IE student?

T13: Industrial Engineering

I: Are you enjoying your classes?

T13: uh. sure yes.

I: What kind of thing are you studying right now?

T13: uh. I am study, I am studying about uh. uh. this call em em MPC it, it means manu, manufacturing, planning control, it's about the uh.(.) how to plan plan before you: like manufacturing

I: Why did you choose here, instead of the other states, or another why US instead of England or Australia?

T13: o.k. first of all uh. I, I, I, I come here because I think the, the, the leader of world is here is United States of America so I come here because I think the, the main technology or any: any stuff I can find from this country

I: uh-huh why here?

T13: uh. because (.) first of all, I come here because I, I would like to start at this university and I would like to apply into uh. many university and and in that time I apply to this university too and (.) and the graduate school accept me so I think, I can start here and wait for the reply from another university it's, it's too late so I, I skip it

I: When you first came here and started school, how did you adjust your study habits?

T13: uh. ah. (.) in that time $I I$ ' $m I$ ' $m$ very unlike I very nervous uh. in how can I schedule the time but when I study about one one term and I find the re-I can solve this problem, I can figure out how can I (.) how do do the time table like like I can survive (.) here

I: $\quad$ So do you feel you have adjusted to the way of study here?

T13: a lot

I: When you first came to the United States, what surprised you?

T13: uh. the culture first thing is culture and technology, uh-huh some-sometime something that I cannot find ah. like technology like uh. or or culture some some culture like a refund refund thing in Thailand not no no refund or technology I cannot find some (.) uh. like uh. something that mo very modern cannot find like computer or communication something like that

I: $\quad$ Can you tell me about some tradition that you have in Thailand like here we celebrate Halloween, Christmas's day, Valentine's day.

T13: You mean the day, right the day,

I: well, what kind of celebrations, traditions that you celebrate

T13: o.k. like Song Kran, Song Kran is mean happy New Year in Thailand but it's not on the January first, it's on the like uh-huh. ap April thirteen uh-huh

I: and what happens then? 
T13: It's like, on the time, it, it is a summer time so everybody play uh. water, play water and like (.) throw it to another person very enjoy uh-huh

I: $\quad$ So you like this holiday

T13: yes, uh-huh

I: In your country, do you think there is any corruption?

T13: Sure (.) I think so. Many I think every every not not every country I mean uh. most (.) in in in every country they have good guy and bad guy and (.) for exam, for example Japan you you know they they they have corruption too so it it's the same in every country they have should have corruption.

I: $\quad$ Can you give some example.

T13: uh-huh. like (.) when when we (.) when we when we want (.) when the the government want the construct like the the the railway or (.) is called the monorail and sometime it's hard something wrong, something weird in in in the the investment or something so $I I I$ heard many new many many rumor that I don't know what it's true or it's just rumor.

I: If there's anything you can do to solve the problem?

T13: I don't think so because I am just a student (.) if I like if I know both and and I think I if I am I on the high position, I can solve (.) some problems

I: What are you most proud of in your country?

T13: ah. the most the most thing that I proud it is ah. he is the the king of Thailand uh-huh. Bhumipon he he is the bes-he is I he he is the most respect (.) in Thailand uh. because he like god you know like god but he is not exactly god he is like every people expect him and he he can do the something good for some when we have like (.) ah-huh chaos on in in my country he can stop immediately he can stop the chaos immediately no country can do that only Thailand can do that I'm very surprised and and and many country surprise too why what happen only he come to said stop every was stop 
I: $\quad$ Please tell me your name and then tell me a little bit about what you're studying?

T19: o.k. my name is and my last name is uh. I have a nick name that people most most people call me by ___ and I'm studying Food Science um. my, I'm gonna be focusing on uh. sensory evaluation of food I: Are you almost finished with your study?

T19: No, I just started yeah this, this is my second term because I'm uh. doing Ph.D actually I finish my Master $u$ in summer I defended in summer

I: here?

T19: yeah, here, yeah, I mean, I just started Ph.D in fall, this is my second quarter

I: How long have you been in school in the United States?

T19: uh. I have been in a city (.) I, I was in there for like um. one not one year eight, eight months yeah and I got accepted here so I been here for two and a half years

I: If you can remember when you first started studying in the United States, did you have to change your study habits from what you were used to?

T19: um. I, when I was, when I was in Thailand, I mean after I graduated from university and I study language for like six months yeah and I came here and I start uh. I started my my English school here in Portland, yeah. I change pretty much because it's more pressure and yeah it's in English so it's more difficult

I: Why did you choose the United States to go to school, instead of another English-speaking country and why did you choose here?

T19: I chose here because I like here long time ago already I heard a lot about here, how green it is and but I never heard about how much rain it has so $I I$ like here long time ago and because $I I$ like to study Food Science yeah I graduated my Master degree in Food Science and I like to continue on on Food Science so this university is pretty good in Food Science that's why I chose it here and I heard that it's very peaceful and I chose USA to come for English (.) I would think that because because I am aiming for here for my future um. ah. study that's why I came to USA for English.

I: good choice

T19: (laugh)

I: When you first came to the United States, what surprised you the most?

T19: What surprise me? I had a lot of culture shock that um. that um. yeah. people people are nice people were nicer than I thought that they they were gonna be because I think that could be because just it's in here because when I went to other states like especially a big city I didn't like it at all people were not nice but people in here are very nice and people can talk to you even they don't know you at all and that that would I think that would surprise me most. People just "hi, how are you?", "how you's your school today?" da da da da and I don't know you what you talking yeah but that's that's a pretty good impression 
I: Why do you think it would be so different between two states, here and there right next to each other?

T19: I don't know, I would think that people and I mean like (.) I would think that it's because of in there could be more people and more competitive. People don't care much about each other but here the environment and everything just so relaxing, yeah. people I would guess that, that's one of the reason that people here are nicer than people in there

I: What are you the most proud of in Thailand?

T19: um. my parents yeah. you mean like (.)

I: That's fine, in your country, whatever.

T19: ah. in my country you mean, I would say that I'm proud of our culture Thai culture is I would think one of the most interesting and most beautiful culture in the world yeah, I like seniority in Thailand which we don't see much here and Thailand people respect the older people all the time and that, that keep, I think that keep the the society peaceful and nice to live

I: What do you dislike most about America?

T19: dislike most food (laugh) I hate American food, sorry to say that but yeah just burger some just something that not creative actually and just yeah that's what I hate most because Thai food is very good and very tasty but American food all taste the same

I: really? I think some American cooks are very creative but maybe you don't see.

T19: yeah. maybe yeah. because I mostly I go to like restaurant and that's not really uh. authentic American restaurant and a lot of burgers, a lot of sandwiches and those those yeah I, I just don't like it at all.

I: $\quad$ You ever watched any of those food programs on TV like the food of [an untranscribable word]

T19: once in a while and most of them are like uh. Italian food and other countries' food not American food (laugh)

I: Does America have any food?

T19: I, that' what I was gonna ask that what is American food, besides burger, what is American food?

I: Yeah, I think it's a very good question, "what is American food?" Well, that's kind of lead us to the next question, tradition. There are a lot of American traditions, we celebrate particularly holidays like Halloween, Valentine's day. Can you tell me about some celebration or tradition that you enjoy.

T19: In Thailand, I enjoy the most is Chinese New Year because my family is Chinese so we don't care much about American, I mean international New Year but for Chinese New Year we have big celebration, have a lot of food and yeah. everybody get together just like family union, reunion on that day so yeah. I have fun most on that day

I: $\quad$ Is that different from Thai New Year? 
T19: ah. it is, yeah it's different from Thai New Year, actually I would guess that Thai people would enjoy Thai New Year the most because it's a long weekend and they can go out and have so many activities going on but my family is Chinese and my my grandfather is very very strict in like Chinese tradition everything so II never been allow to go out to play or to do some activities like other other kids so I didn't enjoy it at all (laugh) just keep watching they have fun it's not fun

I: Oh. now that brings us to another question. Many students who have come here to the United States experience differences that they have in their home like not not living with their parents here and they have a lot more freedom what to do, do you think that will influence their cultural attitude when they go home?

T19: yes. definitely I would think yes but ah. I would think that it depends on on individual because if the people that come here and the kids that come here if they are mature enough I mean (.) not physically like mentally or yeah. they they are mature I don't think that's gonna uh. change much but if they are still like young kids like just graduated from high school they don't have really firm ah. (.) belief or or any firm any strong feeling about their own culture they could be changed easily

I: Would that make it more difficult when they go back home?

T19: when they go back home, they will have another culture shock (laugh) at home, yeah. but I, I think that they would stick with their own group the people that are similar to them like materialistic or whatever that they are and they won't I think their gonna change the the they won't be changed because of the Thai culture but they gonna change the uh. they gonna change Thai culture in the future if these kinds of kids have more and more and they become majority yeah. we gonna loose our culture one day.

I: Since you brought that up, do you have any proverbs that you can think of that help you maintain your value here?

T19: um. no, I don't have any speci, specific proverb I just but I just have, how to say (.) belief and I I'm just proud of what I am what I have been before so and I see that what is not right I think what is not right I'm not gonna take it in my in my thinking could be right for some other people but if it's not for me not for my family not for my society I'm not taking it.

I: What do you think some of those value are?

T19: ah. materialistic that is something that's very obvious that a lot of small kids from Thailand that just came and they were not that that materialistic before but when they came here and they become more materialistic it could be influence from other other friends from other countries that they really spend their lives like spend money or anything like nothing but yeah and another thing could be ah. the feeling of uh. seniority they may not feel like senior people are someone that they need to respect anymore. 
APPENDIX D

BREAKDOWN BY SUBGROUP 


\section{APPENDIX D}

\section{BREAKDOWN BY SUBGROUP}

BREAKDOWN BY NUMBER OF WORDS PER MINUTE $\mathrm{t}$-tests for Independent Samples of Group

Filled Pauses

$\begin{array}{lcccc}\text { Variable } & \text { Number of Cases } & \text { Mean } & \text { SD } & \text { SE of Mean } \\ \text { Filled pauses } & & & & \\ \text { wpm }<120 & 11 & 9.9454 & 5.409 & 1.631 \\ \text { wpm }>120 & 9 & 6.9365 & 2.443 & .814 \\ - & & \end{array}$

Mean Difference $=3.0089$

Levene's Test for Equality of Variances: $f=1.491 p=.238$

t-test for Equality of Means

\begin{tabular}{lcrccc} 
Variances & t-value & df & 2-Tail Sig & SE of Diff & $95 \%$ CI for Diff \\
\hline Equal & 1.54 & 18 & .141 & 1.954 & $(-1.097,7.115)$ \\
Unequal & 1.65 & 14.48 & .120 & 1.823 & $(-.889,6.906)$
\end{tabular}

Unfilled Pauses

\begin{tabular}{|c|c|c|c|c|}
\hline Variable & Number of Cases & Mean & SD & SE of Mean \\
\hline Unfilled pauses & & & & \\
\hline wpm $<120$ & 11 & 6.0620 & 2.989 & .901 \\
\hline $\mathrm{wpm}>120$ & 9 & 2.2989 & 1.600 & .533 \\
\hline
\end{tabular}

Mean Difference $=3.7631$

Levene's Test for Equality of Variances: $f=3.314 p=.085$ t-test for Equality of Means

\begin{tabular}{|c|c|c|c|c|c|}
\hline Variances & t-value & $\mathrm{df}$ & 2-Tail Sig & SE of Diff & $95 \%$ CI for Diff \\
\hline Equal & 3.39 & 18 & $* .003$ & 1.110 & $(-1.431,6.095)$ \\
\hline Unequal & 3.59 & 15.81 & .002 & 1.047 & $(-1.541,5.985)$ \\
\hline
\end{tabular}


Retraced Restarts

\begin{tabular}{lcccc}
\hline Variable & Number of Cases & Mean & SD & SE of Mean \\
\hline Retraced restarts & & & & \\
wpm $<120$ & 11 & 8.5945 & 3.975 & 1.199 \\
wpm $>120$ & 9 & 5.6415 & 3.027 & 1.009 \\
& & &
\end{tabular}

Mean Difference $=2.9530$

Levene's Test for Equality of Variances: $f=.390 \mathrm{p}=.540$

t-test for Equality of Means

\begin{tabular}{llrrrr} 
Variances & t-value & df & 2-Tail Sig & SE of Diff & $95 \%$ CI for Diff \\
\hline Equal & 1.83 & 18 & .083 & 1.611 & $(-.432,6.338)$ \\
Unequal & 1.88 & 17.94 & .076 & 1.567 & $(-.340,6.246)$
\end{tabular}

Unretraced Restarts

\begin{tabular}{lrrrr}
\hline Variable & Number of Cases & Mean & SD & SE of Mean \\
\hline Unretraced restarts & & & & \\
wpm $<120$ & 11 & 1.5231 & 1.219 & .367 \\
wpm $>120$ & 9 & 1.0999 & .569 & .190
\end{tabular}

Mean Difference $=.4232$

Levene's Test for Equality of Variances: $f=1.149 p=.298$ t-test for Equality of Means

\begin{tabular}{lccccc} 
Variances & $\mathrm{t}$-value & $\mathrm{df}$ & $2-\mathrm{Tail}$ Sig & SE of Diff & $95 \%$ CI for Diff \\
\hline Equal & .96 & 18 & .351 & .442 & $(-.506,1.353)$ \\
Unequal & 1.02 & 14.73 & .323 & .413 & $(-.459,1.306)$
\end{tabular}


Sum of the Occurrences of Four features

\begin{tabular}{lcccc} 
Variable & Number of Cases & Mean & SD & SE of Mean \\
\hdashline Sum & & & & \\
wpm $<120$ & 11 & 26.1250 & 6.215 & 1.874 \\
wpm $>120$ & 9 & 15.9768 & 4.413 & 1.471 \\
- & &
\end{tabular}

Mean Difference $=10.1482$

Levene's Test for Equality of Variances: $f=.921 p=.350$

t-test for Equality of Means

\begin{tabular}{lccccr} 
Variances & t-value & df & 2-Tail Sig & SE of Diff & 95\% CI for Diff \\
\hline Equal & 4.11 & 18 & $* .001$ & 2.466 & $(4.967,15.330)$ \\
Unequal & 4.26 & 17.71 & .000 & 2.382 & $(5.138,15.159)$
\end{tabular}

WPM

\begin{tabular}{lcccc}
\hline Variable & Number of Cases & Mean & SD & SE of Mean \\
\hdashline wpm & & & & \\
wpm $<120$ & 11 & 104.4192 & 12.462 & 3.757 \\
wpm $>120$ & 9 & 138.7394 & 18.594 & 6.198
\end{tabular}

Mean Difference $=-34.3202$

Levene's Test for Equality of Variances: $f=2.962 p=.102$

t-test for Equality of Means

\begin{tabular}{lccccc} 
Variances & t-value & df & 2-Tail Sig & SE of Diff & $95 \%$ CI for Diff \\
\hline Equal & -4.93 & 18 & $* .000$ & 6.962 & $(-48.947,-19.693)$ \\
Unequal & -4.74 & 13.50 & .000 & 7.248 & $(-49.919,-18.721)$
\end{tabular}


Semantic WPM

\begin{tabular}{lcrrr} 
Variable & Number of Cases & Mean & SD & SE of Mean \\
\hdashline wpm & & & & \\
wpm $<120$ & 11 & 81.8161 & 8.925 & 2.691 \\
wpm $>120$ & 9 & 116.3875 & 17.982 & 5.994 \\
\hdashline
\end{tabular}

Mean Difference $=-34.5714$

Levene's Test for Equality of Variances: $f=6.152 p=.023$

t-test for Equality of Means

\begin{tabular}{lccccc} 
Variances & t-value & df & 2-Tail Sig & SE of Diff & $95 \%$ CI for Diff \\
\hline Equal & -5.61 & 18 & $* .000$ & 6.162 & $(-47.518,-21.625)$ \\
Unequal & -5.26 & 11.19 & .000 & 6.570 & $(-49.003,-20.140)$
\end{tabular}

Mann-Whitney U - Wilcoxon Rank Sum W Test

by Group

Filled Pauses

$\begin{array}{lrl}\text { Mean Rank } & \text { Cases } & \\ 11.82 & 11 & \text { Group }=.0 \quad \mathrm{wpm}<120 \\ 8.89 & 9 & \text { Group }=1.0 \mathrm{wpm}>120 \\ & 20 & \text { Total }\end{array}$

\begin{tabular}{|c|c|c|c|}
\hline \multirow[t]{2}{*}{ U } & \multirow[t]{2}{*}{ W } & \multirow{2}{*}{$\begin{array}{l}\text { Exact } \\
\text { 2-Tailed p }\end{array}$} & Corrected for Ties \\
\hline & & & $\mathrm{z} \quad$ 2-Tailed $\mathrm{p}$ \\
\hline 35.0 & 80.0 & .2947 & -1.1016 \\
\hline
\end{tabular}




\begin{tabular}{|c|c|c|c|c|c|}
\hline \multirow{5}{*}{$\begin{array}{l}\text { by Group } \\
\text { Mean Rank } \\
13.82 \\
6.44\end{array}$} & \multicolumn{4}{|c|}{ Unfilled Pauses } & \\
\hline & \multirow{2}{*}{\multicolumn{2}{|c|}{$\begin{array}{c}\text { Cases } \\
11\end{array}$}} & & \\
\hline & & & \multicolumn{2}{|c|}{ Group $=.0 \mathrm{wpm}<120$} & \\
\hline & & 9 & \multicolumn{2}{|c|}{ Group $=1.0 \mathrm{wpm}>120$} & \\
\hline & & 20 & Total & & \\
\hline \multirow[t]{2}{*}{$\mathrm{U}$} & \multirow[t]{2}{*}{ W } & & \multirow{2}{*}{$\begin{array}{l}\text { Exact } \\
\text { 2-Tailed p }\end{array}$} & \multicolumn{2}{|c|}{ Corrected for Ties } \\
\hline & & & & $\mathrm{z}$ & 2-Tailed $p$ \\
\hline \multirow[t]{2}{*}{13.0} & 58.0 & & .0042 & -2.7730 & $* .0056$ \\
\hline & \multicolumn{5}{|c|}{ Retraced Restarts } \\
\hline \multicolumn{6}{|l|}{ by Group } \\
\hline Mean Rank & & Cases & & & \\
\hline 12.45 & & 11 & \multicolumn{3}{|c|}{ Group $=.0 \mathrm{wpm}<120$} \\
\hline \multirow{2}{*}{8.11} & & 9 & \multicolumn{3}{|c|}{ Group $=1.0 \mathrm{wpm}>120$} \\
\hline & & 20 & Total & & \\
\hline \multirow[t]{2}{*}{$\mathrm{U}$} & \multirow[t]{2}{*}{$\mathrm{W}$} & & \multirow{2}{*}{$\begin{array}{l}\text { Exact } \\
\text { 2-Tailed p }\end{array}$} & \multicolumn{2}{|c|}{ Corrected for Ties } \\
\hline & & & & & 2-Tailed p \\
\hline 28.0 & 73.0 & & .1119 & -1.6334 & .1024 \\
\hline
\end{tabular}

by Group

Mean Rank

Unretraced Restarts

11.73

Cases

9.00

11 Group $=.0 \mathrm{wpm}<120$

9 Group $=1.0 \mathrm{wpm}>120$

20 Total

\begin{tabular}{|c|c|c|c|c|}
\hline \multirow[t]{2}{*}{$\mathrm{U}$} & \multirow[t]{2}{*}{ W } & \multirow{2}{*}{$\begin{array}{c}\text { Exact } \\
\text { 2-Tailed p }\end{array}$} & \multicolumn{2}{|c|}{ Corrected for Ties } \\
\hline & & & $\mathrm{z}$ & 2-Tailed p \\
\hline 36.0 & 81.0 & .3312 & -1.0256 & .3051 \\
\hline
\end{tabular}


Sum of the

Occurences of Four Features

by Group

$\begin{array}{ccc}\text { Mean Rank } & \text { Cases } & \\ 14.41 & 11 \quad \text { Group }=.0 \mathrm{wpm}<120 \\ 5.72 & 9 \quad \text { Group }=1.0 \mathrm{wpm}>120 \\ & 20 \quad \text { Total }\end{array}$

\begin{tabular}{|c|c|c|c|c|}
\hline \multirow{2}{*}{$\mathrm{U}$} & \multirow[t]{2}{*}{ W } & \multirow{2}{*}{$\begin{array}{c}\text { Exact } \\
\text { 2-Tailed } p\end{array}$} & \multicolumn{2}{|c|}{ Corrected for Ties } \\
\hline & & & $\mathrm{z}$ & 2-Tailed p \\
\hline 6.5 & 51.5 & .0004 & -3.2681 & ${ }^{*} .0011$ \\
\hline
\end{tabular}

\section{WPM}

by Group

Mean Rank

Cases

$\begin{array}{rrl}6.00 & 11 & \text { Group }=.0 \mathrm{wpm}<120 \\ 16.00 & 9 & \text { Group }=1.0 \mathrm{wpm}>120 \\ & 20 & \text { Total }\end{array}$

\begin{tabular}{|c|c|c|c|c|}
\hline \multirow[t]{2}{*}{$\mathrm{U}$} & \multirow[t]{2}{*}{ W } & \multirow{2}{*}{$\begin{array}{l}\text { Exact } \\
\text { 2-Tailed p }\end{array}$} & \multicolumn{2}{|c|}{ Corrected for Ties } \\
\hline & & & $\mathrm{z}$ & 2-Tailed $p$ \\
\hline .0 & 144.0 & .0000 & -3.7621 & $* .0002$ \\
\hline
\end{tabular}

by Group

Mean Rank

Semantic WPM

$\begin{array}{rrl}6.00 & 11 & \text { Group }=.0 \quad \mathrm{wpm}<120 \\ 16.00 & 9 & \text { Group }=1.0 \mathrm{wpm}>120 \\ & 20 & \text { Total }\end{array}$

\begin{tabular}{|c|c|c|c|}
\hline \multirow[t]{2}{*}{$\mathrm{U}$} & \multirow[t]{2}{*}{ W } & \multirow{2}{*}{$\begin{array}{l}\text { Exact } \\
\text { 2-Tailed } p\end{array}$} & Corrected for Ties \\
\hline & & & 2-Tailed p \\
\hline .0 & 144.0 & .0000 & -3.7607 \\
\hline
\end{tabular}


APPENDIX E

A SAMPLE BROCHURE 


\section{APPENDIX E}

\section{A SAMPLE BROCHURE}

A brochure should include the following ideas for Thai graduate students who will intend to study in the United States. These ideas may help improve their motivation in developing fluency.

1. Keep in mind that your primary reason in coming to the United States(besides attainment of your educational goals) is developing proficiency in the English language) Seize the opportunity at least once or twice a day to converse in L2 with English native speakers. Do not limit your English conversations to classroom participation and discussion with your professors. Conversations in social settings, markets, shopping malls or even telephone calls to the electric company to inquire about a bill will benefit you in terms of accumulating a wider range of vocabulary. Research (by Gass \& Varonis, 1994) indicates that interaction leads you to noticing English native speakers' production and your own production. For example, you may restructure your grammar, sentence structure, or vocabulary because differences between your English and that spoken by English native speakers are noticed.

2. Be open to opportunities in a pro-active way and be outgoing. Do not cling to Thai friends and speak only Thai language. Integrate yourselves into this new culture, enjoy it and try to experience as many new things as possible. Remind yourself that your 
stay in the United States is limited to only a few years, and the remainder of your life will be spent in Thailand. When you cling to Thai friends all of the time, you are more likely to yield the speaking floor to the most fluent speaker in your group. That person will always be the one who communicates better, while you lose the opportunity to practice. You may feel embarrassed about speaking English in front of your Thai friends. Remember, no one is perfect, and there is nothing to be ashamed about. In an effort to reduce clannishness with your Thai friends, try to find at least one American friend that you can talk to at a conversational level other than just normal greetings. This will provide you with ample opportunity to speak and learn English. As Gardner and Lambert (1972) suggest, there are two types of motivation for L2 learners. The integrative motivation orients toward L2 learners whose intention is to immerse themselves into the target culture of its people; "The student wishes to learn more about the other cultural community because he is interested in it in an open-minded way, to the point of eventually being accepted as a member of that other group" (Gardner \& Lambert, 1972, p.3). There is a relationship between integrative motivation and the necessity to communicate (Spolsky, 1977). The more a person desires to be one of the members of the target culture, the greater he needs to learn the language. On the other hand, the instrumental motivation orients L2 learners who study the language toward a career or other practical reasons (Gardner \& Lambert, 1972). It is found that integrative motivation is essential to successful attainment of the advanced levels of L 2 proficiency. 
3. Try to make yourselves as active learners rather than passive learners even though it might not suit your personality. Give yourselves a try. Bear in mind that you will spend a lot of money studying here. Seliger (1977) described learners who are eager to put themselves into the position of interaction initiator in order to use the opportunity to talk as high input generators (HIG). Conversely, learners who do not grab practice opportunities to talk, neither in formal nor natural settings are considered as low input generators (LIG). For instance, in the language classroom, LIG barely participate unless the teacher specifically calls on them; whereas, HIG are actively involved by "calling out, answering out of turn, and working out answers to questions or drill cues directed to other student" (Seliger, 1977, p.266). These behaviors lead input to be directly focused on HIG. As a result, HIG develop their language skills faster and probably qualitatively better than LIG.

4. Learning style in the United States is different from Thailand. Participation in class is expected. It might be very difficult to speak up in class for the first time; but if you try, it will get easier. In order to share the knowledge with your classmates or professors, be a risk taker, attempt to get your intended messages across. Keep four tips in mind from Ely's experiment (1986) that students' voluntary classroom participation is a positive sign for language learners:

1) Do not hesitate to try out "using a newly encountered linguistic element"(p.8).

2) Be willing to express complicated or difficult ideas in $L 2$.

3) Do not mind making mistakes or looking foolish in your attempts to use the language.

4) Tend to rehearse the new sentences silently before trying to speak them. 
These characteristics reflect the speakers' intentions to use the language and seek out practice opportunities for themselves. Seliger (1977) finds that learners "who intensively interact with their language environment" (p.275) are more likely to be "higher achievers" (p.267) than those who do not. 
APPENDIX F

A WORKSHOP FOR ESL INSTRUCTORS:

TEACHING FLUENCY 


\section{APPENDIX $F$}

\section{A WORKSHOP FOR ESL INSTUCTORS:}

\section{TEACHING FLUENCY}

The study makes us realize the importance of fluency. ESL classroom is one of the places for students to have opportunities to develop fluency. Insufficient practice speaking English will result in reduced fluency. Fluency should be included in a lesson plan for speaking portion of ESL class. What ESL teachers might consider to do is to first introduce briefly the notion of fluency and its features (see Chapter II). Then, explain reasons of the occurrences of non-fluency problems such as speakers' lack of vocabulary, thinking of what to say next and making several attempts to express the ideas, and the inability to communicate the thoughts in L2 that match with the L1. This study suggests solutions to non-fluency behaviors. One of the solutions is to teach students applicable strategies, for example, vocabulary strategies, lengthening etc. (see details in p.72). Two activities suggested partially by Smith et al., 1992 were: 1) have interactive activities in class such as break students into pairs. Each student has a list of five words from teachers. Tell them to use vocabulary strategies to get their partners to say the exact words on the list, and give themselves scores. Finally, select only two teams that have the highest scores. The whole class will have chances to listen to those two groups competing and notice how they use those strategies. 2) Another activity 
(Smith et al., 1992) is to assist students thinking in English and use strategies to eliminate hesitation and repairs. First, divide the whole class into groups of three or four. Each member of the group will take one of the topics provided by the teacher and talk about for 2 minutes without stopping. Have a timekeeper in every round. The topic should be difficult or unfamiliar for students to elaborate upon such as bed, liar, weather, the letter "C" etc. After that, the other members give feedback how well he/she did on strategies in counterbalancing non-fluency. 